\title{
Global Analysis of The Signalling Network of Breast Cancer Cells In Response To Progesterone.
}

Roni H. G. Wright ( $\nabla$ roni.wright@crg.es )

Barcelona Institute of Science and Technology (BIST) https://orcid.org/0000-0002-8194-4614

Viviana Vastolo

Barcelona Institute of Science and Technology (BIST)

Javier Quilez Oliete

Barcelona Institute of Science and Technology (BIST)

Jose Carbonell-Caballero

Barcelona Institute of Science and Technology (BIST)

Miguel Beato

Barcelona Institute of Science and Technology (BIST)

\section{Research Article}

Keywords: Progesterone, breast cancer, chromatin, signal transduction, cell cycle regulation, PARylation, phosphorylation, nuclear structure, kinases, transcription factors

Posted Date: December 15th, 2021

DOI: https://doi.org/10.21203/rs.3.rs-958950/v2

License: (c) (i) This work is licensed under a Creative Commons Attribution 4.0 International License.

Read Full License 
1 Global Analysis of the Signalling Network of Breast Cancer Cells in Response to

\section{Progesterone.}

4 Roni H. G. Wright ${ }^{1,2^{*}}$ Viviana Vastolo ${ }^{1}$, Javier Quilez Oliete ${ }^{1}$, José Carbonell-

5 Caballero $^{1}$, and Miguel Beato ${ }^{1,3 *}$

$7{ }^{1}$ Center for Genomic Regulation (CRG), Barcelona Institute of Science and 8 Technology (BIST), Dr. Aiguader 88, Barcelona 08003, Spain

92 Basic Sciences Department, Faculty of Medicine and Health Sciences, Universitat 10 Internacional de Catalunya, Barcelona, Spain.

$11{ }^{3}$ Universitat Pompeu Fabra (UPF), Barcelona, 08003 Spain.

13 *corresponding authors: roni.wright@crg.eu; miguel.beato@crg.eu

17 Background: Breast cancer cells enter into the cell cycle following progestin exposure

18 by the activation of signalling cascades involving a plethora of enzymes, transcription

19 factors and co-factors that transmit the external signal from the cell membrane to 20 chromatin, ultimately leading to a change of the gene expression program. Although 21 many of the events within the signalling network have been described in isolation, how 22 they globally team up to generate the final cell response is unclear.

23 Methods: In this study we used antibody microarrays and phosphoproteomics to reveal 24 a dynamic global signalling map that reveals new key regulated proteins and phosphor25 sites and links between previously known and novel pathways. T47D breast cancer cells 
were used, and phosphosites and pathways highlighted were validated using specific antibodies and phenotypic assays. Bioinformatic analysis revealed an enrichment in novel signalling pathways, a coordinated response between cellular compartments and protein complexes.

31 Results: Detailed analysis of the data revealed intriguing changes in protein complexes 32 involved in nuclear structure, epithelial to mesenchyme transition (EMT), cell 33 adhesion, as well as transcription factors previously not associated with breast cancer 34 proliferation. Pathway analysis confirmed the key role of MAPK following 35 progesterone and additional hormone regulated phosphosites were identified. Full 36 network analysis shows the activation of new signalling pathways previously not associated with progesterone signalling in breast cancer cells such as ERBB and TRK. As different post-translational modifications can mediate complex crosstalk mechanisms and massive PARylation is also rapidly induced by progestins, we provide details of important chromatin regulatory complexes containing both phosphorylated and PARylated proteins.

Conclusions: This study contributes an important resource for the scientific community, as it identifies novel players and connections meaningful for breast cancer cell biology and potentially relevant for cancer management.

Keywords

49 Progesterone, breast cancer, chromatin, signal transduction, cell cycle regulation, 


\section{Background}

54 Female steroid hormones, oestrogen and progesterone play a key role not only in the normal development of target tissues during puberty, pregnancy and menopause but also in breast and endometrium cancer cell proliferation. Breast cancer cells respond to progestin exposure with two intermingled pathways that culminate in extensive gene expression changes and entry in the cell cycle. The classical view is that the hormone diffuses through the cell membrane and binds to intracellular progesterone receptors (PR), which are maintained in an inactive state by a chaperone complex, including Heat Shock Proteins 70 and 90 (HSP70/90). Upon hormone binding, PR weakens its interaction with the chaperones, dimerizes and moves to chromatin where eventually binds to palindromic DNA sequences called progesterone responsive elements (PREs) (Pina et al., 1990). Once bound to chromatin, PR recruits various co-regulators and chromatin remodellers that modulate access for the transcription machinery including RNA polymerase II (Beato et al., 1995).

This simplified model was completed later by the finding that a tiny fraction of PR (3-5\%) is attached to the cell membrane via palmitoylation at C820 (Migliaccio et al., 1998; Pedram et al., 2007), forming a complex with estrogen receptor alpha (ERa) (Ballare et al., 2003). Upon binding progestins, the membrane anchored PR activates SRC, either directly (Boonyaratanakornkit et al., 2001) or via ERa, initiating a kinase signalling pathway that ends in activation of the extracellular signal-regulated kinase (ERK) (Ballare et al., 2003). ERK1 phosphorylates intracellular PR at S294 favouring its dissociation from the chaperone complex. In the cell nucleus ERK1 activates MSK1 (Mitogen-and stress activated protein kinase 1), resulting in the formation of a ternary 
complex of PR-ERK1-MSK1, which is the active form of PR able to regulate chromatin structure and gene expression. ERK also activates cyclin dependent kinase 2 (CDK2) that in turns activates ARTD1 (ADP-ribose transferase 1) by phosphorylating two serines in the NAD+ binding pocket (Wright et al., 2012). Phosphorylation contributes to dissociation of histone $\mathrm{H} 1$ and $\mathrm{H} 2 \mathrm{~A} / \mathrm{H} 2 \mathrm{~B}$ dimers (Vicent et al., 2006; Wright et al., 2012) and to local chromatin opening by further recruitment of transcription factors, co-regulators, histone modifiers (PCA, P3000) and ATP-dependent chromatin remodellers (NURF and BAF), ultimately leading to the activation of gene expression changes (Vicent et al., 2011; Vicent et al., 2009a; Vicent et al., 2010). Moreover, there is also evidence for the activation by progesterone of other signaling pathways induced by progesterone, such as AKT (Fu et al., 2010), cAMP (Garg et al., 2017; Takahashi et al., 2009), GSK3 (Rider et al., 2006) and STAT (Hagan et al., 2013). However, many of these studies use different cells of a different types of cells derived from endometrial or ovarian tissues (Lee and Kim, 2014; Wang et al., 2007).

In addition to the key role of the kinase cascades, we have identified a pivotal role for another post-translational modification in progestin induced gene regulation; namely Poly-ADP-ribosylation (PARylation). As discussed above the PAR polymerase PARP1, also known as ADP-ribosyltransferase 1 (ARTD1), is activated within the initial minutes following hormone exposure via phosphorylation by CDK2 (Wright et al., 2012), giving rise to a large increase in PARylation within the cell nucleus (Wright et al., 2012). Parylation of ARTD1 itself and of chromatin proteins is essential for the initial dissociation of histone H1 (Nacht et al., 2016; Vicent et al., 2016). We also found that degradation of PAR to ADP-Ribose by PAR glycohydrolase (PARG) is required for complete chromatin remodelling and activation of the gene expression network (Wright et al., 2016). Mass spec analysis of the proteins interacting with PAR in T47D 
cells exposed to progestins revealed structural proteins, DNA damage response proteins

102 and chromatin modifying enzymes (Wright et al., 2016). One key enzyme identified in

103 this study was NUDT5 or NUDIX5 (Nudix hydrolase 5), which hydrolyses ADPR to

104 AMP and ribose-5-phosphate. Subsequently, we found that upon dephosphorylation at

105 T45, NUDT5 can use ADPR and diphosphate for the synthesis of ATP (Wright et al.,

106 2016). In this way, part of the ATP consumed during the synthesis of $\mathrm{NAD}^{+}$and stored

107 in PAR is recovered and used for chromatin remodeling and changes in gene

108 expression. The synthesis of nuclear ATP is transient, peaking at 40 minutes after

109 hormone exposure and returning to basal levels after $\sim 60$ min. However, although we

110 know that nuclear ATP synthesis is essential for the initial chromatin remodeling, the

111 role of the nuclear ATP at later time points is unclear. We can envision several

112 hypotheses; as a direct, local source of ATP for the massive amount of ATP-dependent

113 chromatin remodelling and 3D conformational changes induced by hormone (Le Dily

114 et al., 2019; Vicent et al., 2011) or to facilitate phase separation of chromatin fiber

115 (Wright et al., 2019). In any case, we know that nuclear ATP synthesis by NUDT5 is

116 essential for the generation and maintenance of the cancer stem cell population (Pickup

117 et al., 2019).

118 Over the past years, there has been a large number of studies investigating the

119 role and mechanism of action of one or more of the pathway components in response

120 to progesterone exposure, revealing a dynamic crosstalk between canonical pathways

121 (Boonyaratanakornkit et al., 2008; Faivre et al., 2005; Qiu et al., 2003; Skildum et al.,

122 2005). However, these studies focused on one or very few components at a time, and

123 do not explain how these various pathways interact and coordinate the cell response to

124 hormone. The work described here aims to provide a more comprehensive map of 125 progesterone signalling in breast cancer cells, combining antibody arrays technology, 
126 shotgun proteomics, and previously published PARylation datasets to develop for the

127 first-time a global map of the dynamic signalling events induced by progestins in breast

128 cancer cells.

129

130 Methods

131 Cell culture

132 The hormone receptor positive breast cancer cell line $\mathrm{T}_{47 \mathrm{D}}^{\mathrm{M}}$ (CLS Cat\# 133 300353/p525_T-47D, RRID:CVCL_0553) was used in all experiments unless

134 otherwise stated. T47D ${ }^{\mathrm{M}}$ cells were routinely grown in RPMI (Supplemented with 10\% 135 fetal bovine serum (FBS), penicillin/streptomycin (pen/strep), L-glutamine (L-glut) as 136 previously described (Wright et al., 2016). For hormone induction experiments, cells 137 were seeded at a concentration of $5 \times 10^{6}$ per $150 \mathrm{~mm}$ cell culture dish in RPM1 white 138 (15\% charcoal stripped FBS, Pen/strep, L-glut) for 48 hours. 16 hours prior to hormone 139 induction (10nM R5020), medium was replaced with RPM1 white (0\% FBS, Pen/strep, 140 L-glut). Samples were harvested at the time points indicated.

142 BCA Assay

143 The total protein content of the samples was calculated prior to antibody array, mass

144 spec or western blotting analysis using BCA assay (Thermo Fisher, catalogue number 145 23227) according to manufactures instructions.

Protein Visualisation

148 Changes in phosphorylation sites within individual proteins identified was confirmed

149 by western blotting as previously described (Nacht et al., 2016) using specific 150 antibodies; Progesterone receptor (PGR) phospho-S162 (Abcam Cat\# ab58564, 
151 RRID:AB_883089), and as a loading control, total PGR (Santa Cruz Biotechnology

152 Cat\# sc-7208, RRID:AB_2164331), total CDK2 (Santa Cruz Biotechnology Cat\# sc153 6248, RRID:AB_627238) or CDK2 phospho-T160 (Abcam Cat\# ab47330, 154 RRID:AB_869087).

155

156 Antibody Microarray

157 Phosphorylation antibody array analysis was carried out by Kinexus ${ }^{\mathrm{TM}}$ using Kinexus

$158{ }^{\mathrm{TM}}$ Antibody Microarray (KAM) technology. For each time point 3 biological replicates

159 were prepared independently. For each replicate, 50ug of protein lysate was prepared

160 and samples prepared by Kinexus ${ }^{\mathrm{TM}}$ in house (Kinexus Bioinformatics Corporation,

161 RRID:SCR_012553). Signal quantification was performed using ImaGene 8.0

162 (ImaGene, RRID:SCR_002178) from BioDiscovery (BioDiscovery,

163 RRID:SCR_004557). Background corrected raw intensity data was logarithmically

164 transformed with base 2 and $Z$ scores calculated (Cheadle et al., 2003). Any poor-

165 quality spots based on morphology and/or background, were flagged as unreliable and

166 removed from any subsequent analysis.

167

168

Mass Spec Sample Preparation 


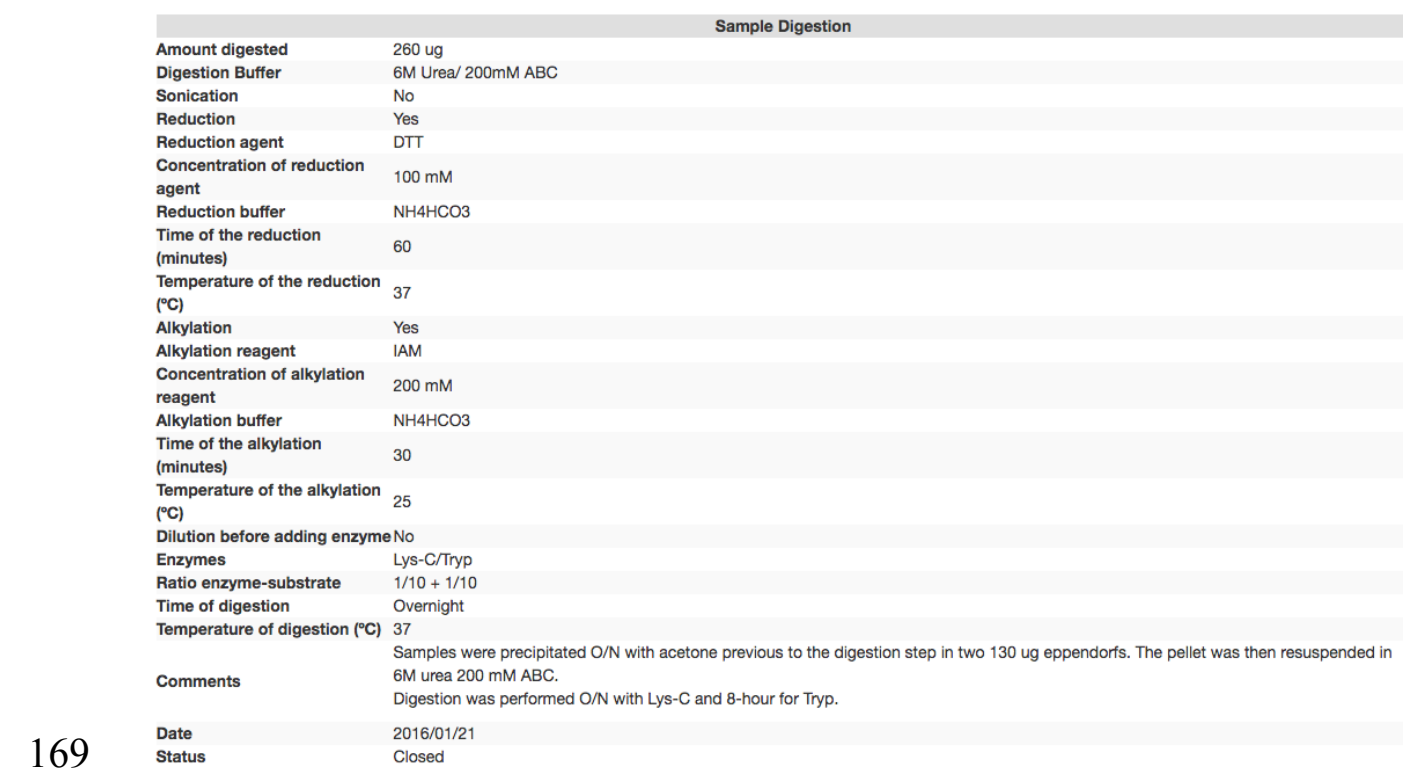

170 Bioinformatic Procedures

171 Gene Ontology (GO) GO-Biological process (GO-BP), GO-Molecular Process (GO-

172 MF), KEGG (KEGG, RRID:SCR_012773) and Biocarta (BioCarta Pathways,

173 RRID:SCR_006917) pathway analysis of networks was carried out using GeneMania

174 application (GeneMANIA, RRID:SCR_005709) (Warde-Farley et al., 2010) within

175 Cytoscape (Cytoscape, RRID:SCR_003032). Clustering analysis, similarity analysis

176 was carried out using GeneE. Network analysis was performed using Cytoscape v3.5

177 (Shannon et al., 2003). The initial prior knowledge network (PKN) was generated based

178 on known protein-protein interactions only validated experimentally. Significantly

179 enriched pathways were analyzed within the network using CytoKEGG application

180 within Cytoscape. The parent network and each of the individual pathway networks are

181 available for visualization and further analysis using following cytoscape session link

182 found within supplementary materials. Comprehensive resource of mammalian protein

183 complexes (Corum analysis) was carried out using online tool http://mips.helmholtz-

184 muenchen.de/corum/ CORUM, RRID:SCR_002254 (Giurgiu et al., 2018). Functional

185 classification GO biological process (BP), molecular function (MF) and cellular

186 component (CC) were carried out using molecular signatures database (MSigD) within 
187 Gene Set Enrichment (GSEA) tool (Gene Set Enrichment Analysis, 188 RRID:SCR_003199) and terms with a $\mathrm{p}$ value of less than 0.001 were considered 189 significantly enriched (Liberzon et al., 2011; Subramanian et al., 2005).

Kaplan Meyer and Protein Expression in Clinical Samples

193 Analysis of the overall survival of breast cancer patients using a Kaplan-Meier plot

194 were carried out using KMPlotter (Gyorffy et al., 2010), https://kmplot.com/analysis/

$195 \mathrm{n}=3951$. All patients' samples were included in the analysis shown, i.e ER, PR status,

196 subtype, lymph node status and grade. Analysis of protein expression levels in breast

197 tumour versus normal samples were representative of those within the Human Protein 198 Atlas database (Uhlen et al., 2015) http://www.proteinatlas.org.

\section{Results}

\section{Prior Knowledge Network}

203 Before starting to add quantitative dynamic data to the already existing knowledge of

204 progesterone signalling events in breast cancer cells, we have generated a "prior 205 knowledge network (PKN)", based on the published literature (Fig.S1A-B, and 206 Supplementary material; Cytoscape Session 1). Each protein-protein interaction is 207 characterized based on type (interaction, phosphorylation or dissociation) and is 208 displayed as a unique edge. The corresponding literature is given in Fig. S1B and within 209 the Cytoscape session. The PKN already shows the key role played by kinases in the 210 response of breast cancer cells to progestins. First, progestins via ERa activate SRC1 211 that phosphorylates MAPKK1, that activates ERK1, that phosphorylates PGR, 
212 resulting in dissociation from the HSP90A and B proteins (Haverinen et al., 2001;

213 Smith, 1993). Activated ERK1 also phosphorylates ERa at S118 (Kato et al., 1995).

214 ERK1 in association with hormone receptors translocates to the cell nucleus where it

215 phosphorylates MSK1 (Reyes et al 2016), leading to the formation of an active complex

216 PR-ERK-MSK1 that interacts with chromatin containing accessible PRE. Activated PR

217 also interacts with PLK1 that activates MLL2 (Wierer et al., 2013), with CDK2 that

218 phosphorylates and activates ARTD1 (Wright et al., 2012), and with JAK2 that

219 activates STAT5 (Hagan et al., 2013). Simultaneously, membrane activated SRC1, also

220 activate RAS and EGFR (Boonyaratanakornkit et al., 2007), which feeds back

221 activating the MAPK cascade. Membrane associated ERa also activates PI3K and

222 cAMP, which upon binding with AKT and PKA respectively lead to the activation (via

223 interaction and direct phosphorylation) of GSK3, mTOR (Ciruelos Gil, 2014; Ortega et

224 al., 2020) and the arginine methyltransferases CARM1 and PRMT1 within the nucleus

225 (Lange, 2008; Li et al., 2003; Malbeteau et al., 2020). This brief description of the PKN

226 shows that it already encompasses a great degree of complexity and complementary

227 connection that need additional data to be resolved.

2. Microarrays of antibodies to phosphorylated sites in proteins

230 Our plan was to combine antibody microarray technology and shotgun

231 phosphoproteomics in $\mathrm{T}_{47 \mathrm{D}^{\mathrm{M}}}$ breast cancer cells exposed to $10 \mathrm{nM}$ R5020 for different

232 lengths of time, as previously described (Wright et al., 2012). For each experimental

233 approach and exposure time total protein extracts were harvested in triplicate. For the

234 antibody arrays, data was collected, filtered for quality control and summarized as $\log _{2}$

235 ratio over time zero (as described in materials and methods, Fig. S2A). This dataset

236 provided 246 unique phosphorylation sites corresponding to 155 proteins (Fig. 1A, 
237 Supplementary Table S1). The majority of proteins contain 1 phosphorylation site, 238 although for several proteins (Tau, RB1, MAP2K1, PTK2 and the protein kinase 239 RPS6KA1) 7 or more significantly regulated phosphorylation sites were identified (Fig. 240 S2B).

241 Analysis of the number of phosphorylation sites clearly showed a rapid 242 activation already 1-minute following hormone (68 significant phosphorylation events 243 Fig 1B). Signalling persists throughout the time course showing two peaks at 30- and 244 360-minutes following hormone (Fig. 1B). Phosphorylation sites were characterized as 245 up or down-regulated, using a threshold for the $\log _{2}$ fold change with respect to time 0 246 of $-0.6<$ or $>0.6$ respectively (Fig 1C). We see a trend for early phosphorylation sites 247 to be dynamically increased compared to time zero, in contrast to later time points 248 where protein phosphorylation sites as a whole decrease compared to time zero (Fig 249 1C). The majority of phosphorylation sites identified belong to protein kinases (45\%), 250 co-factors (11\%), transcription factors (18\%) and structural proteins (13\%). (Fig 1D). 251 Combining the identified phosphorylation sites over the time course reveals that the 252 majority of sites are regulated at more than one time point (Fig 1E), however the protein 253 function enrichment does not alter significantly over time, with kinases and 254 transcription factors being the main protein groups where the phosphorylation sites are 255 observed (Fig. S2C).

256 Pathway and gene ontology (GO) for biological function (BP) molecular 257 function (MF) analysis revealed a significant increase in Cancer pathways (Fig S2D), 258 signal transduction, biopolymer metabolic process and kinase activity (Fig 2SE and F). 259 Within this dataset we observed a strongly upregulated phosphorylation of the MAPK 260 Signal-Integrating Kinase 1, MNK1 at T250/T255 (Fig. 1F) in T47D in response to 261 progesterone stimulation. Phosphorylation of MNK1 at T250/T255 by ERK induces 
the activity of MNK1 (Dolniak et al., 2008). Once activated, MNK1 phosphorylates its

263 targets, including the proto-oncogene Eukaryotic Translation Initiation Factor 4E

264 (EIF4E), for which we also observed a modest phosphorylation which follows a similar

265 pattern to MNK1 (Fig. 1F). Activation of MNK1 has been shown to promote cell

266 proliferation thus MNK1 inhibitors appear as an exciting opportunity for cancer 267 therapy. MNK1 signalling play a key role in invasive breast cancer growth (Guo et al., 268 2019), MNK1 inhibitors have been shown to block breast cancer proliferation in 269 multiple cell lines (Wheater et al., 2010), and its downstream target EIF4E is 270 overexpressed in tumour versus normal samples from breast cancer patients (Fig. 1G) 271 and associated with a poor overall survival (Fig. 1H). Our results are the first indication 272 that MNK1 activation may be relevant for progesterone induced breast cancer cell 273 proliferation.

We found that CDK2 plays an important role in progesterone signaling, 275 activating ARTD1, and phosphorylating histone H1 (Wright et al., 2012). CDK2 276 activity is controlled by the formation of an active complex with the cyclin partner; 277 either Cyclin E or A. In addition to binding the cyclin partner, CDKs are also controlled 278 via interactions with Kinase Inhibitory Proteins (KIPs). p27/KIP is rapidly 279 dephosphorylated at T187 in response to hormone, dropping sharply at 1 minute after 280 hormone exposure (Fig. 1J), when CDK2 is phosphorylated and activated. 281 Phosphorylation of $\mathrm{p} 27$ at $\mathrm{T} 187$ results in the proteins ubiquitination and degradation 282 and inhibits the interaction with CDK2 (Grimmler et al., 2007), which would result in 283 the release of CDK2 from the inhibitory protein resulting in the activation of ARTD1 284 and subsequent nuclear effects. In addition, we observe the coordinated activation of 285 the upstream kinase of CDK2 at T160; ERK at Y202/204 (Fig. 1K) and could validate 286 the phosphorylation of CDK2 T160 via ERK by western blotting in the presence of 
ERK inhibition (Fig. 1L). CDK2 at T160 is the active phosphorylation site of CDK2 peaking at 1-minute following hormone exposure (Fig. 1K) in contrast to the inactive phosphorylation site of CDK2 (T14/Y15) which peaks at 60 minutes following 290 hormone exposure to silence the kinase (Fig. 1M). The activity of the phosphatase 291 CDC25C is key for the removal of the inhibitory T14/Y15 phosphorylation sites of CDK2. The phosphatase itself is inactivated by phosphorylation at S216. We observe a peak in CDC25C phosphorylation prior to and following 60 minutes of hormone exposure, which would permit the phosphorylation of the inhibitory phosphorylation site in CDK2 (Fig. 1M and N). Going one step further; MAPKAPK2, the kinase which phosphorylates $\mathrm{CDC} 25 \mathrm{C}$ at $\mathrm{S} 216$ is activated following the same time dynamic as its target (Fig. 1O). Although the importance of CDK2 in progestin induced cell proliferation has been studied (Trevino et al., 2016; Wright et al., 2012) the complex mechanism of CDK2 activation; phosphorylation of active/inactive marks, activation and regulation of upstream phosphatases and kinases was not clear until now (Fig. 1P).

301 These examples of the dynamic phosphorylation of MNK1 and CDK2 highlight the insight that can be gained by this type of global signaling datasets.

\section{Shotgun phosphoproteomics}

To complement the microarray dataset, we performed shotgun phosphoproteomic 306 analysis using mass spec. Phospho-peptides from $\mathrm{T} 47 \mathrm{D}^{\mathrm{M}}$ cells exposed to $10 \mathrm{nM}$

307 R5020 for the same duration as in the array experiments, were enriched using TiO2 and 308 phosphorylated peptides identified by LC-MS-MS (Fig. 2A, Fig. S3A, Supplementary 309 Table S1). We identified changes in 310 unique phosphorylation sites within 264 310 unique proteins (Fig 2B and C). The majority of proteins exhibited regulation of a single 311 phosphosite, except for the serine/arginine repetitive matrix protein, SRRM1, involved 
312 in mRNA processing and the TP53 enhancing protein TP53BP1, that exhibited 8 and

31310 regulated phosphorylation sites respectively (Fig. S3B). Most phosphorylation sites

314 identified were phosphor-serine consistent with the biological ratio of residue specific

315 phosphorylation (Fig 2D). Over the time course, changes at each time point were

316 identified as either up $\left(\log _{2} \mathrm{FC}>0.6\right)$ or down $\left(\log _{2} \mathrm{FC}<-0.6\right)$ regulated (Fig. 2E). Up-

317 regulated sites prevailed at early time points and many of these phosphorylation sites

318 were significantly regulated at more than one time point (Fig. 2F). Pathway and GO-

319 BP (Biological Process) and MF (Molecular Function) enrichment analysis was

320 consistent with the antibody array enrichment and revealed an increase in pathways in

321 cancer, biopolymer metabolic process and kinase activity (Fig. S3C-E).

322 PR S294 is rapidly phosphorylated in response to hormone resulting in its 323 activation and dissociation from chaperone complexes and increase protein turnover 324 (Lange et al., 2000). In recent years it has been shown that clinical samples assigned as 325 "PR low" actually have elevated levels of phosphorylated PR S294 and that this 326 phosphorylation is associated with a genetic signature linked to cancer stem cell growth 327 and increased recurrence which may have implications for the treatment of PR low 328 patients with anti-progestins (Knutson et al., 2017). Phosphorylation of PR S162 in the 329 hinge region showed a strong hormone induced increase by mass spec (Fig. 2G and H). 330 Phosphorylation within this region of PGR has been previously reported to be mediated 331 by CDK2 (Knotts et al., 2001), which we were able to confirm as the specific 332 phosphorylation of S162 PR in response to progesterone was strongly decreased in the 333 presence of CDK2 inhibition (Fig. 2H). 
336 In order to investigate the dynamics of progestin signalling over time and with the aim

337 of avoiding inherent biases generated from either technical approach, we combined the 338 significantly regulated phosphorylation sites from both datasets (Fig. 1 and 2) resulting 339 in a list of 420 unique phosphorylation sites within 390 proteins (Fig. S4A). PCA 340 analysis of the samples reveals a clear separation of the phosphorylation data at 6 hours 341 following hormone, given the majority of phosphorylation sites are rapid effect this 342 separation of the latest time point may reveal changes in protein levels at this time point. 343 The majority of these proteins showed the regulation of a single phosphorylation event 344 with the exception of several highlighted proteins, including FAK, MAPT and EGFR 345 (Fig. S4C). As in the individual analysis, phosphorylation sites were significantly 346 regulated over several time points (Fig. S4D) and showed a switch from up-regulated 347 sites early after hormone exposure to down-regulated sites at later time points (Fig. 348 S4D). KEGG pathway analysis shows a significant enrichment in MAPK, PI3K-AKT, 349 neurotrophin (TRK) and ERBB signalling pathways (Fig. 3A, Supplementary Table 350 S2), in addition to pathways key in the progression of cancer, specifically cancer stem 351 cells, such as focal adhesion (Fig. 3A).

352 GO cellular component analysis reveals a dynamic pattern of specific cellular 353 compartments over time (Fig. 3B, Supplementary Table S3). As expected, over the 354 whole-time course, proteins are mainly found within the cytosol and nucleoplasm. 355 However, prior to hormone exposure, phosphorylated proteins are enriched in RNA 356 transcription repression complex and nuclear chromatin. The addition of hormone 357 rapidly induces the phosphorylation of the membrane rafts, components of focal 358 adhesion and protein kinases consistent with published works whereby signalling 359 initiates from the plasma membrane. This transient phosphorylation of the membrane 360 rafts diminish after 1 minute and is followed by the phosphorylation of transcription 
361 factors and proteins within the cytoskeleton (Fig. 3B). Interestingly, in line with our

362 findings showing the generation of nuclear ATP synthesis independent of

363 mitochondrial supplementation at 30 minutes after hormone we observe an enrichment

364 in phosphorylated proteins located within the mitochondrial membrane at 15 minutes

365 (Fig. 3B). The dynamic regulation of these proteins; CYB5B (cytochrome b5), the

366 transcriptional activator ATF2 (Cyclic AMP-dependent transcription factor ATF-2),

367 RPS6KB1 (Ribosomal protein S6 kinase beta-1) and PI4KB (Phosphatidylinositol 4-

368 kinase beta) (Fig. S4F) may suggest an as yet undiscovered crosstalk between the

369 nuclear and mitochondrial ATP synthesis pathways. Mitochondrial PR (PR-M) is a

370 truncated isoform of the nuclear progesterone receptors PRB and PRA, which lacks the

371 N-terminal DNA binding domain present in PRA and PRB but does contain the hinge

372 region responsible for dimerization and the ligand binding domain (Price and Dai,

373 2015). PR-M has been shown to increase cellular respiration hence cell energy levels

374 in response to ligand in various physiological situations and animal models (Dai et al.,

375 2019). Therefore, the coordinated phosphorylation of proteins within the mitochondria

376 in response to ligand (Fig. S4F) in breast cancer cells may provide an interesting insight

377 into a possible crosstalk between mitochondrial PR-M and the nuclear receptors PRA

378 and PRB.

379 At 60 minutes following hormone exposure the main localization of

380 phosphorylation changes and shifts again to nuclear matrix proteins and proteins found

381 within distinct regions of the nucleus, such as PML bodies (Fig. 3B group IV), which

382 may be involved in the reorganization of chromatin in response to progestins (Le Dily

383 et al., 2019). At 6 hours following hormone exposure cells enter the early stages of

384 entering the cell cycle and movement is increased. This is also evident by the 
enrichment of phosphorylation sites in proteins within cell-cell junctions, the cytoskeleton and microtubules (Fig. 3B group V).

\section{Protein class analysis}

389 The majority of identified phosphorylated proteins $(60 \%)$ were assigned to one class, 390 however due to the promiscuous nature of enzymes nearly $40 \%$ were assigned to more 391 than one class (Fig. S4G). Taking first only the parent class into account, we observed 3925 distinct functions; 1) nucleic acid binding, 2) enzymes, 3) structural proteins, 4) 393 protein modulators, and 5) proteins involved in signalling, membrane and cell-cell 394 contacts (Fig. S4H). Each function class consists of sub-groups (Fig. S5A-F). The 395 Nucleic Acid binding class includes DNA binding proteins, helicases, nucleases and 396 RNA binding protein subgroups (Fig. S5A). The enzyme class is dominated by kinases 397 but also includes histone modifying enzymes, hydrolases, ligases and oxidoreductases 398 (Fig. S5D). The structural class is dominated by cytoskeleton proteins (Fig. S5F). The 399 protein modulator class includes chaperones and various kinases and $\mathrm{G}$ proteins 400 regulators (Fig. S5C). The cell signalling and the membrane/cell-cell contact classes 401 are more complex and include many specialized proteins such as signalling molecules, 402 receptors and transporters (Fig. S5B and E).

404 Gene Ontology of Biological Processes (GO-BP) and Molecular Function (GO-MF) 405 showed an enrichment in signal transduction and general biological processes across 406 the entire time course (Fig. S6A and B, Supplementary Tables S4 and S5). However, 407 several interesting dynamic functions were identified. For instance, transcription co408 factors, transcriptional repressors and transcription factor binding were already 409 enriched 1 minute after hormone exposure (Fig. S6B) consistent with our previous 
observations of rapid transcription factor recruitment following hormone exposure

411 (Nacht et al., 2016; Vicent et al., 2011). We observed enrichment in ATP binding and 412 Adenyl-ribonucleotide binding after 5 and $60 \mathrm{~min}$ of hormone exposure (Fig. S6B), 413 which may represent regulation of the two cycles of ATP dependent chromatin 414 modifiers in response to progesterone (Vicent et al., 2009b; Wright et al., 2016). KEGG 415 pathway analysis reveals a significant enrichment in signalling and in many cancer 416 pathways, including Prostate, Glioma, CML, lung, AML, endometrial and pancreatic 417 cancer, as well as focal adhesion and tight junctions (Fig. S6C). Annotated signalling 418 cascades were significantly enriched at all time points in response to progestin, 419 including MAPK, neurotrophin (TRK), ERBB, FC-receptor and insulin signalling (Fig. 420 S6C).

\section{Specific pathways: Roles of AMPK, insulin TNFa, and PIK3}

423 K Means clustering analysis revealed six patterns of regulation over the time course 424 (Fig. 3C). Similarity analysis of all phosphorylation sites within all clusters shows 425 several interesting dynamics. First, "Early-risers" cluster 1 and 4, are positively 426 correlated on the similarity matrix and show their initial increase in phosphorylation 427 early at 1 and 5 minutes, respectively (Fig. 3D). GO-BP analysis of the proteins 428 contained within these clusters shows an enrichment in signal regulation, and signalling 429 cascades including Hippo, NFk-B and MAPK pathways (Fig. 3E, Supplementary Table 430 S9). Second, clusters 3 and 6 show an opposing nature (negative correlation Fig. 3D). 431 This antagonistic behavior of the two clusters is clearly shown averaging the signal of 432 all phosphorylation within each cluster (Fig. 3F). Corum (comprehensive resource of 433 mammalian protein complexes) analysis of the significantly enriched protein 434 complexes contained within clusters 3 and 6 (Supplementary Table S6) showed that 
most protein complexes were enriched in one cluster or the other (Fig. 3G), likely representing crosstalk. Ten protein complexes were found to be enriched in both cluster 3 and 6 , having phosphorylation sites within the same protein complex regulated in an opposite manner (Fig. 3G).

One such complex was the cMyc-ATPase-Helicase complex, which contains 5 440 proteins; cMyc, the chromatin remodeling component BAF53, the ATP-dependent 441 helicases RUVBL1 and 2 (also known as TIP48 and 49) and the histone 442 acetyltransferase, TRRAP. This complex is involved in chromatin organization, histone 443 acetylation and transcriptional regulation (Park et al., 2002). Analysis of the 444 phosphorylation sites showed that two sites (S373, T58) within Myc were increased 445 early after hormone exposure, and decreased after 60 minutes, whereas one site of 446 BAF53 (S233) shows the opposite dynamic (Fig. 3H). Database analysis also reveals a 447 strong overexpression of BAF53 in tumour versus normal samples in multiple cancer 448 types (Fig. 3I). Myc has an important role in breast cancer growth via the activation of 449 AMPK (von Eyss et al., 2015).

The AMP-activated protein kinase (AMPK), exhibited a decrease in T183 451 phosphorylation in response to hormone. This site is phosphorylated by CAMKK1 or 4522 (Hurley et al., 2005). AMPK is a master sensor, and its activation inhibits several 453 kinase pathways including mTOR, NfkB, JAK/STAT, insulin and Hippo (Hadad et al., 454 2008; Montero et al., 2014; Yamaguchi and Taouk, 2020; Zhao et al., 2017). In 455 addition, active AMPK inhibits the phosphorylation of PR S294, PR recruitment to 456 chromatin and the activation of progesterone regulated genes (Wu et al., 2011). 457 Activation of the kinase, specifically requires the phosphorylation of AMPK at T183 458 by CAMKKs, and de-phosphorylation of this site has been shown to be induced by 459 estrogens and androgens in adipocytes (McInnes et al., 2012; McInnes et al., 2006). 
460 Previous published results and the data presented here suggests a model where AMPK 461 must be silenced in order for regulatory pathways described and PR itself to be active, 462 which is what we observe within 1 minute of progesterone stimulation (Fig. 3J and K). Further in-depth analysis of the complexes which are regulated by 464 phosphorylation in response to progestin revealed a full list of complexes with at least 4652 proteins phosphorylated in response to hormone. One of them is the Sam68-p85 466 P13K-IRS-1-IR signalling complex, which encompasses the insulin receptor (INSR), 467 the insulin receptor substrate 1 (IRS1), the $\mathrm{KH}$ domain containing transduction468 associated protein 1 (Sam68) and the phosphatidylinositol 3-kinase regulatory subunit 469 alpha (GRB1). This protein complex is involved in insulin signalling and has been 470 proposed to provide a link between the PI3K pathway and other signalling cascades of 471 insulin or p21/RAS (Sanchez-Margalet and Najib, 2001). We observed a dynamic 472 phosphorylation of several sites within the complex (Fig. 4A), including 4 distinct 473 phosphorylation events within IRS1, two of which peak at 1 minute (S312, S639) and 474 two sites where the peak in phosphorylation is observed at 60 minutes (Y1179, and 475 Y612). S312 has been shown to be directly phosphorylated by c-Jun N-terminal kinase 476 (JNK1) in breast cancer signalling (Mamay et al., 2003) and this phosphorylation 477 inhibits its interaction with IKKA. S639 is phosphorylated by mTOR has been linked 478 to PI3K/Akt/mTOR signalling in breast cancer (Eto, 2010; Tzatsos, 2009) and effects 479 the intracellular localization of IRS1 (Hiratani et al., 2005). Y1179 has been reported 480 to be phosphorylated by IGF1R or INSR itself (Xu et al., 1995) and Y612 481 phosphorylation activates the interaction with PIK3R1 (Valverde et al., 2003). The phosphorylation of several components of the TNFa/NFkB signalling 483 complex were also identified (Fig. 4B). This complex is involved in I-kB kinase/NF$484 \mathrm{kB}$ signalling in tumour progression. Indeed, complex components IKK $\alpha$, RelB and 

p52 are associated with decreased cancer-specific survival in ERa-positive breast cancer (Paul et al., 2018). This may be linked to the cancer stem cell niche, which we showed recently was present in T47D cells grown in 3D cultures (Pickup et al., 2019). NFkB regulates self-renewal in breast cancer stem cell (BCSC) models and deletion of IKK $\alpha$ in mammary-gland epithelial cells affects progestin-driven breast cancer 490 (Schramek et al., 2010; Shostak and Chariot, 2011). Indeed, the upstream activator 491 RANK ligand (RANKL) and hence the RANK pathway promotes mammary tumor 492 formation, (Gonzalez-Suarez et al., 2010), (Schramek et al., 2010). Another example is 493 the P130Cas-ER-cSrc-PIK3 kinase complex (Fig. 4C). Which has been shown to 494 induce transcriptional changes in response to estrogen and mammary proliferation in 495 breast cancer. The authors showed that estradiol triggers the association of ERa, c-Src, 496 the p85 subunit of PI 3-kinase (PI3K) and p130Cas in a macromolecular complex and 497 activates the c-Src kinase leading to p130Cas-dependent Erk1/2 phosphorylation 498 (Cabodi et al., 2004; Cabodi et al., 2006). Given the similarity of the phosphorylation 499 dynamics, peaking early at 5 and 15 minutes across Src, PIK3 and ESR1 within the 500 complex induced by progestin (Fig. 4C right panel) this may (similarly to the induction 501 by estrogen shown by others) present a novel ERa-ERK-cSrc activation mechanism in response to progestin in breast cancer cells.

\section{Crosstalk between Progestin induced Phosphorylation and PARylation}

506 Recently, it has been shown that the majority of PARylation events on eukaryotic 507 nuclear proteins take place on serine residues rather than acidic residues as previously 508 accepted (Bonfiglio et al., 2017a; Bonfiglio et al., 2017b; Leidecker et al., 2016; Liu et 509 al., 2017; Martello et al., 2016; Messner et al., 2010). Given the enrichment of serine 
in the phosphorylation dataset (Fig. 2D) and the importance of both PTMs in progestin

511 gene regulation (Wright et al., 2016), we investigated the overlap between PARylation

512 sites and phosphorylation sites within protein complexes. We identified 52 proteins, 513 which were both phosphorylated and PARylated in response to progestins in breast 514 cancer cells (Fig. 4D). Cellular component analysis of this set of 52 proteins indicates 515 a significant enrichment in nuclear, cytoskeleton and chromatin contained proteins 516 (Fig. 4E, Supplementary Table S7), in line with the well described role of PAR in the 517 nucleus, nuclear organization, chromatin organization, relaxation and transcriptional 518 regulation (Hassa et al., 2006; Hoch and Polo, 2019; Leung, 2014; Thomas and Tulin, 519 2013). This finding may indicate a crosstalk between PARylation and phosphorylation 520 with regards to nuclear structure and chromatin organization. Analysis of complexes 521 significantly enriched within this group of proteins revealed 12 protein complexes 522 (Supplementary Table S8), which contained proteins both PARylated and 523 phosphorylated. One of them is the KSR1-RAF1-MEK complex composed of MEK1 524 and 2, both PARylated and phosphorylated, and RAF1 which is phosphorylated (Fig. 525 4F). This complex is involved in the MAKPKKK cascade, and in response to EGF it 526 activates BRAF mediated phosphorylation of MEK1, at 3 sites, and MEK2, which 527 activate MAPK1 and 3 (McKay et al., 2009). In our dataset we observe a clear change 528 in phosphorylation of all members of the complex in response to progestin (Fig. 4F 529 right panel).

530 We also observed the phosphorylation and PARylation of the Emerin complex 531 (Fig. 4G). This complex is involved in DNA replication, transcription and structural 532 integrity of the nucleus, specifically of the inner nuclear membrane (Holaska and 533 Wilson, 2007). Depletion of Emerin results in changes in the organisation and dynamics 534 of the nucleus, increased chromatin mobility and a mis-localisation of chromosome 
territories (Ranade et al., 2019). Within this complex we find proteins phosphorylated, PARylated, or phosphorylated and PARylated (Fig. 4G). Given the role of PAR in the structure of the nucleus, this complex may present and interesting example for studying the PARylation, phosphorylation crosstalk.

As discussed, prior to hormone exposure PR is present in an inactive complex with the HSP70 and 90 proteins as part of the Kinase Maturation Complex. We know that progestins promote the phosphorylation and dimerization of the receptor and we found that phosphorylation of the HSP90 and 70, along with other members of the complex, is initiated within 1 minute of hormone exposure (Fig. 4H), again showing a rapid and concerted phosphorylation of several members of the complex (Fig. 4H). In addition, the HSPs are also PARylated as compared to other components where only phosphorylation (MARK2, MAP2K5) or PARylation (14-3-3 components) are present

(Fig. 4I and J). Further investigation regarding the crosstalk between PARylation and phosphorylation within protein complexes will be the focus of future studies.

\section{Progesterone Signalling Network Generation}

In order to understand the crosstalk between the signalling pathways activated by Progesterone in breast cancer cells, a Protein-Protein Interaction (PPI) network was generated using all identified phosphorylation sites (Supp. File: Network session 2), based on known PPI (evidence based). The resulting network consists of 427 nodes (proteins) and 4309 unique interactions (edges) (Fig S7A). Pathway analysis was carried out on this network, using Genemania ${ }^{\mathrm{TM}}$, and 23 statistically significant $(p<0.01)$ pathways were identified (Supplementary Table S9 and S10). The proteins and interactions (nodes and edges) associated with each pathway were selected and new 
networks generated (Supp. File: Network session 2). Several of which are shown in Fig. S7B-G and discussed briefly below.

One such pathway, the Fc receptor signalling pathway was identified as enriched (Fig. S6C) and the PPI network is shown in Fig. S7B. Fc receptors are cell surface proteins that recognize the FC fragment of antibodies, mainly on immune cells. However, recent studies have shown that different subsets of Fc receptors may play a role in tumour cells (Nelson et al., 2001). In particular, it was shown that T47D cells express the FcyRI (CD64) These FC-receptor expressing breast cancer cells can activate the tyrosine kinase signal transduction pathway. Indeed, T47D cells treated with selective tyrosine kinase inhibitors do not proliferate in a FC receptor- tyrosine kinase signalling dependent manner (Nelson et al., 2001).

As mentioned before (Fig. S6C), another pathway identified as activated in response to progestin is the ERBB-EGF network (Fig. S7D and H). ERBB2 (HER2) is overexpressed in $15-20 \%$ of breast cancer in response to EGF activation, and plays a major role in EMT (Elizalde et al., 2016). PR interacts with ERBBs and induces the translocation of ERBB2-PR-STAT3 complex to the nucleus. ERBB2 acts as a coactivator of STAT3 and drives the activation of progestin regulated genes, especially genes such as Cyclin D1 that do not contain a HREs (Beguelin et al., 2010; Hsu and Hung, 2016). Blocking PR signalling in PR-ERBB2 positive breast cancer patients has been suggested as a treatment (Proietti et al., 2009). The ERBB pathway may represent a new mechanism for further study to understand the activation of these "non-classical" PR dependent genes in response to progestin. In addition to the role of ERBB2, the role of ER activation in response to progesterone in breast cancer cells is also critical, as shown in Fig. 4C we observe the coordinated activation of the ESR1-Src-PIK3 complex peaking at 15 minutes following hormone exposure. The phosphorylation site of ESR1 
which increases is S104. ER S104 phosphorylation is essential for ER activity (Thomas et al 2008) and it has been suggested that hyperphosphorylation of ER at these sites may contribute to resistance to tamoxifen in hormone receptor positive breast cancer (Leeuw et al 2011, Jeffreys et al 2020, Skliris et al 2010). ER S104 phosphorylation by ERK has been shown previously in response to estrogen and EGF but not progesterone exposure. In addition, ER S104 has been implicated in mTOR signaling (Alayev et al 2016). Given the phosphorylation of ER, the dynamic activation of the ER membrane complex and the role of mTOR in AMPK and insulin signalling described earlier (Fig. $3 \mathrm{~J}$ and $4 \mathrm{C}$ ) this phosphorylation site may present a key step in the cellular response to progesterone in breast cancer.

A pathway exhibiting strong activation by progestins is the Insulin signalling major role in normal mammary gland development and R5020 has been shown to induce the expression of insulin receptor substrate-2 in MCF7 cells (Cui et al., 2003a;

598 Cui et al., 2003b). Moreover, IGF signalling via IRS2 is known to be essential for breast 599 cancer cell migration. It has also been shown that R5020 pretreatment followed by IGF 600 stimulation increases binding of IRS to PI3K-p85 regulatory complex, which in turn 601 activates ERK and AKT signalling (Ibrahim et al., 2008). Interestingly, not only do we 602 observe the activation of the insulin pathway in network analysis (Fig. S7F, but the 603 coordinated phosphorylation of all members of the IRS-PIK3 complex was also 604 identified (Fig. 4A), indicating that indeed progesterone stimulation of breast cancer 605 cells activates the not only the insulin pathway but the coordinated regulation of 606 complexes within it.

\section{Discussion}


The data presented in this paper provides a source of knowledge for the scientific

610 community with regards to progesterone induced gene expression, and the signalling

611 pathways involved. We have shown the rapid induction of phosphorylation using two

612 distinct technologies (Fig. 1 and 2). Pathway analysis showed a strong enrichment in

613 pathways associated with cancer, known and novel Pg-dependent signalling events

614 (Fig. 3A). But also identified signalling pathways not previously known to mediate 615 progesterone action in breast cancer cells, such as MNK1/EIF4E pathway and the 616 connection between CDK2, Cdc25 and the MAPK pathway.

617 Cellular component analysis confirmed our expectations and the statistically 618 significant activation of the cell membrane within 1 minute of hormone exposure (Fig. 619 3B), but also revealed a consistent (over all members) phosphorylation peak within 620 proteins associated within the mitochondria at 15 minutes after hormone exposure (Fig. $6213 \mathrm{~B}$ and S4E). Mitochondrial activation in response to progesterone in breast cancer 622 cells has not been extensively studied yet. Indeed, ATP synthesis 45-60 minutes after 623 hormone stimulation is independent of mitochondrial involvement (Wright et al., 624 2016). However, there are some interesting findings in the literature. Following the 625 observation that the PR negative cell line MCF10A exhibits a progestin-induced cell 626 proliferation (Kramer et al., 2006). Behera and colleagues showed that MCF10A 627 respond to R5020 with an increase in mitochondrial activation (Behera et al., 2009). 628 Given the absence of the nuclear PR in these cells they hypothesized that the activation 629 of progestin-induced cell growth was due to non-genomic metabolic effects, mediated 630 by a yet undiscovered receptor. We propose that the observed mitochondrial activation 631 in T47D in response to progestin (Fig. 3B and S4E) suggests the existence of a third 632 and interconnected hormonal signal transduction pathway via the mitochondria 633 (Demonacos et al., 1996; Hatzoglou and Sekeris, 1997). 
635 of phosphorylation events which follow a similar time response; such as early risers, 636 sustained or late (Fig. 3C). Similarity analysis of these dynamic phosphorylation sites 637 reveals some interesting crosstalk between protein complexes not previously identified 638 as players in progesterone signalling in breast cancer cells (Supplementary Table S6), 639 and complexes where a mobilization of phosphorylation (showing similar dynamics) 640 was observed within the whole macromolecular complex; such as PIK3, NFkB (Fig. $6414 \mathrm{~B}$ and $\mathrm{C})$.

642 Overlap of phosphorylation sites with existing PARylation, revealed 52 proteins 643 for which both phosphorylation and PARylation was found. The data also clearly shows 644 an enrichment in protein complexes that play a role in the structural organisation of the 645 nucleus (Fig. 4D and E), specifically the Emerin complex and Lamin (Fig. 4G). The 646 key location of these complexes at the nuclear membrane, suggests that perhaps these 647 two PTMs may affect and play a role in the dynamic structure of the nucleus. This could 648 be explored in the future by global chromatin proximity $\mathrm{Hi}-\mathrm{C}$ experiments. The 649 complexes identified in this study and the dual post translational modification of 650 proteins with known important roles within the cell may provide exciting opportunities 651 for future studies which aim to understand the crosstalk between Serine PARylation 652 and phosphorylation in the context of nuclear architecture, signalling and breast cancer 653 progression (Supplementary Table S7).

654 In addition to pathway analysis at the single network level (Fig. S7), wherein 655 we identified pathways such as insulin, Fc-receptor and ERBB signalling, it is also 656 clearly important to consider the connection between pathways and networks as a 657 whole. One such example, is the connections between the phosphorylation events 658 within the cytoskeleton, membrane raft and proteins associated with cell adhesion. We 
observe phosphorylation events in multiple proteins within both cell adhesion and the

660 membrane raft, forming tight strongly connected PPI networks (Fig. 5A). A network

661 merge of these two pathways reveals 4 key proteins which are present in both (JAK2,

662 SRC1, LYN and KDR), indicating a strongly connected network (Fig. 5B), which in 663 addition to common members exhibits a large first neighbour selection between the two 664 initial pathways (selection of only direct PPI) (Fig. 5B right panel) with a similar 665 phosphokinetic pattern (Fig. 5C). Incorporation of the significantly enriched 666 phosphorylated proteins within the cytoskeleton (Fig. 3B, Fig. S6D, Supplementary 667 table S3) into the merged network (Fig. 5B) results in a larger global connected network 668 (Fig. 5D) which supports the activation within the membrane proteins after 1-minute 669 following hormone exposure that triggers the subsequent cascades of phosphorylation 670 in the cytoskeleton (Fig. 5E). These findings clearly show the importance of studying 671 the pathways not in isolation, but rather in connection with each other.

672 Other examples of connectivity are observed between the ERK subgroup and 673 the MAPK cascades and the FC Receptor and TRK signalling. ERK and MAPK form 674 a strong network (Fig. 6A and B). As discussed, earlier Fc-receptor signalling shows a 675 strong activation (Fig. S7B). The tropomyosin receptor tyrosine kinases (TRKs) are 676 primarily known for their roles in neuronal differentiation and survival. However, 677 increasing evidence shows that TRK receptors can be found in a host of mammalian 678 cell types to drive several cellular responses (Huang and Reichardt, 2003; Reichardt, 679 2006) Aberrations in TRK signalling, which can occur through events such as protein 680 overexpression, alternative splicing, or gene amplification, can lead to disease such as 681 cancer (Jin, 2020; Meng et al., 2019; Regua et al., 2019). The receptor tyrosine kinase 682 NTRK2, activates GRB2-Ras-MAPK cascade in neurons and increases secretion by 683 epithelial cells in culture in response to estrogen or progestin treatment and NTRK2 
was identified as differentially expressed between stromal and epithelial breast cells

685 which may have implications in invasion and metastasis (Wang et al., 2020) Merging 686 of Fc-receptor and TRK signalling pathways (Fig. 6C) shows a strong protein overlap 687 and a dense connected network with FOXO1 at the center (Fig. 6D). and FOXO1 is 688 phosphorylated after 1 to 5 minutes of progestin exposure (Fig. 6E). Phosphorylation 689 of FOXO1 by PKB/Akt has been shown to be important for the binding to 14-3-3 690 proteins on chromatin (Dobson et al., 2011; Pennington et al., 2018; Tzivion et al., 691 2011; Tzivion and Hay, 2011). The role of these two pathways in progesterone induced 692 gene regulation has not been shown previously. FOXO factors have a key role to play 693 in tumour resistance to therapy and patient outcome (Bullock, 2016). Interestingly, 694 from a clinical perspective, stratifying patients based on either the expression levels of 695 NTRK2 (TRKB) or FOXO1 is predictive of a good prognosis (overall survival) in 696 breast cancer, similar to prognosis based on PR expression (Fig. 6F and G).

697 The examples described here in addition to other examples contained within the data 698 for future discovery show the importance of network connectivity in trying to understand not only individual proteins or pathways but the significant overlap between 700 the pathways within the signalling network activated by progesterone in breast cancer.

701 Further analysis of the detected connections and identification of the key regulators 702 may provide a source of targets for drug discovery aiming at the treatment of hormone 703 receptor positive breast cancer patients.

\section{Conclusions}

706 The data presented here, reveals a high level of complexity in progesterone signalling 707 in breast cancer cells, shedding new light on known proteins and signalling pathways. 708 Functional analysis reveals the activation of known pathways such as MAPK cascade 
709 but also the activation of signalling cascades not previously associated with 710 progesterone signalling such as TRK, TNFa and ERBB. Our analysis indicates that

711 there is a full cellular coordinated response, with proteins activated in different cellular 712 compartments at different times following hormone exposure, in addition to the 713 activation of whole protein complexes previously not associated with progesterone 714 signalling. We believe that this signalling network and the phosphosites identified 715 represent a rich resource for the breast cancer research community, opening up new 716 lines of research and ideas for possible drug discovery projects for the benefit of breast 717 cancer patients.

\section{List of abbreviations}

721 AKT: AKT serine/threonine kinase 1

722 ARTD1: ADP-ribosyltransferase diphtheria toxin-like 1

723 ATP: Adenosine tri-phosphate

724 BAF: BRG1-associated factor 53A

725 BCA: Bicinchoninic acid

726 CAMKK1: Calcium/calmodulin-dependent protein kinase kinase 1

727 CDK1/2: Cyclin dependent kinase

728 EMT: Epithelial to mesenchyme transition

729 ER: Estrogen receptor

730 ERBB: Receptor tyrosine-protein kinase erbB-2

731 ERK1: Extracellular signal-regulated kinase 1

732 KH: K homology

733 MNK1: MAP kinase signal-integrated kinase 1 
734 MSK: Mitogen and stress activated protein kinase 1

735 NUDT5: Nucleoside diphosphate-linked moiety X motif 5

736 NURF: Nucleosome-remodeling factor subunit

737 PAR: Poly-ADP-ribose

738 PARG: Poly-ADP-ribose glycohydrolase

739 PARP1: Poly-ADP-ribose polymerase

740 PCA: Principle component analysis

741 PGR : Progesterone Receptor

$742 \quad$ PKN : Prior knowledge network

743 PML: Prommyelocytic leukaemia

744 PPI: Protein protein interaction

745 PRE: Progesterone responsive element

746

747

Declarations

750 Ethics and approval and consent to participate

$751 \quad$ Not applicable

752

753 Consent for publication

754 Not applicable

755

756 Availability of data and materials

757 The datasets used and analysed during the current study are included in this published 758 article within supplementary tables (Supp Table 1). 


\section{Competing Interests}

761 The authors declare that they have no competing interests.

\section{Funding}

764 This research was supported by European Research Council (Project "4D Genome" 765 609989), the Ministerio de Economía y Competitividad (Project G62426937) and 766 the Generalitat de Catalunya (Project AGAUR SGR 575). European Research 767 Council -Proof Of Concept (Project "Impacct" 825176).

768

769

770

771

772 773

\section{4}

775

781 Ballare, C., Uhrig, M., Bechtold, T., Sancho, E., Di Domenico, M., Migliaccio, A., 782

\section{Authors contributions}

Experimental design; R.H.G.W and M.B. Bioinformatic Analysis; J. Q. O., J.C.C and R.W. Manuscript writing and editing; R.H.G.W and M.B. Experiments; R.W. "All authors read and approved the final manuscript."

\section{Acknowledgements}

We acknowledge the support of all members of the Chromatin, Gene Regulation laboratory and members of the Gene Regulation Cancer and Stem Cells department at Centre for Genomic Regulation (CRG, Barcelona Spain).

\section{References}

Auricchio, F., and Beato, M. (2003). Two domains of the progesterone receptor interact 
with the estrogen receptor and are required for progesterone activation of the c-Src/Erk pathway in mammalian cells. Mol Cell Biol 23, 1994-2008.

Beato, M., Herrlich, P., and Schutz, G. (1995). Steroid hormone receptors: many actors in search of a plot. Cell 83, 851-857.

Beguelin, W., Diaz Flaque, M.C., Proietti, C.J., Cayrol, F., Rivas, M.A., Tkach, M., Rosemblit, C., Tocci, J.M., Charreau, E.H., Schillaci, R., et al. (2010). Progesterone receptor induces ErbB-2 nuclear translocation to promote breast cancer growth via a novel transcriptional effect: ErbB-2 function as a coactivator of Stat3. Mol Cell Biol $30,5456-5472$.

Behera, M.A., Dai, Q., Garde, R., Saner, C., Jungheim, E., and Price, T.M. (2009). Progesterone stimulates mitochondrial activity with subsequent inhibition of apoptosis in MCF-10A benign breast epithelial cells. Am J Physiol Endocrinol Metab 297, E1089-1096.

Bonfiglio, J.J., Colby, T., and Matic, I. (2017a). Mass spectrometry for serine ADPribosylation? Think o-glycosylation! Nucleic Acids Res 45, 6259-6264.

Bonfiglio, J.J., Fontana, P., Zhang, Q., Colby, T., Gibbs-Seymour, I., Atanassov, I., Bartlett, E., Zaja, R., Ahel, I., and Matic, I. (2017b). Serine ADP-Ribosylation Depends on HPF1. Mol Cell 65, 932-940 e936.

Boonyaratanakornkit, V., Bi, Y., Rudd, M., and Edwards, D.P. (2008). The role and mechanism of progesterone receptor activation of extra-nuclear signaling pathways in regulating gene transcription and cell cycle progression. Steroids 73, 922-928. 
804 Boonyaratanakornkit, V., McGowan, E., Sherman, L., Mancini, M.A., Cheskis, B.J., 805 and Edwards, D.P. (2007). The role of extranuclear signaling actions of progesterone 806 receptor in mediating progesterone regulation of gene expression and the cell cycle. 807 Mol Endocrinol 21, 359-375.

808 Boonyaratanakornkit, V., Scott, M.P., Ribon, V., Sherman, L., Anderson, S.M., Maller, 809 J.L., Miller, W.T., and Edwards, D.P. (2001). Progesterone receptor contains a proline810 rich motif that directly interacts with $\mathrm{SH} 3$ domains and activates c-Src family tyrosine 811 kinases. Mol Cell 8, 269-280.

812 Bullock, M. (2016). FOXO factors and breast cancer: outfoxing endocrine resistance. 813 Endocr Relat Cancer 23, R113-130.

814 Cabodi, S., Moro, L., Baj, G., Smeriglio, M., Di Stefano, P., Gippone, S., Surico, N., 815 Silengo, L., Turco, E., Tarone, G., et al. (2004). p130Cas interacts with estrogen 816 receptor alpha and modulates non-genomic estrogen signaling in breast cancer cells. J 817 Cell Sci 117, 1603-1611.

818 Cabodi, S., Tinnirello, A., Di Stefano, P., Bisaro, B., Ambrosino, E., Castellano, I., 819 Sapino, A., Arisio, R., Cavallo, F., Forni, G., et al. (2006). p130Cas as a new regulator 820 of mammary epithelial cell proliferation, survival, and HER2-neu oncogene-dependent 821 breast tumorigenesis. Cancer Res 66, 4672-4680.

822 Cheadle, C., Cho-Chung, Y.S., Becker, K.G., and Vawter, M.P. (2003). Application of $823 \mathrm{z}$-score transformation to Affymetrix data. Appl Bioinformatics 2, 209-217.

824 Ciruelos Gil, E.M. (2014). Targeting the PI3K/AKT/mTOR pathway in estrogen 825 receptor-positive breast cancer. Cancer Treat Rev 40, 862-871. 
826 Cui, X., Lazard, Z., Zhang, P., Hopp, T.A., and Lee, A.V. (2003a). Progesterone 827 crosstalks with insulin-like growth factor signaling in breast cancer cells via induction 828 of insulin receptor substrate-2. Oncogene 22, 6937-6941.

829 Cui, X., Zhang, P., Deng, W., Oesterreich, S., Lu, Y., Mills, G.B., and Lee, A.V. 830 (2003b). Insulin-like growth factor-I inhibits progesterone receptor expression in breast 831 cancer cells via the phosphatidylinositol 3-kinase/Akt/mammalian target of rapamycin 832 pathway: progesterone receptor as a potential indicator of growth factor activity in 833 breast cancer. Mol Endocrinol 17, 575-588.

834 Dai, Q., Likes, C.E., 3rd, Luz, A.L., Mao, L., Yeh, J.S., Wei, Z., Kuchibhatla, M., 835 Ilkayeva, O.R., Koves, T.R., and Price, T.M. (2019). A Mitochondrial Progesterone 836 Receptor Increases Cardiac Beta-Oxidation and Remodeling. J Endocr Soc 3, 446-467.

837 Demonacos, C.V., Karayanni, N., Hatzoglou, E., Tsiriyiotis, C., Spandidos, D.A., and 838 Sekeris, C.E. (1996). Mitochondrial genes as sites of primary action of steroid 839 hormones. Steroids 61, 226-232.

840 Dobson, M., Ramakrishnan, G., Ma, S., Kaplun, L., Balan, V., Fridman, R., and 841 Tzivion, G. (2011). Bimodal regulation of FoxO3 by AKT and 14-3-3. Biochim 842 Biophys Acta 1813, 1453-1464.

843 Dolniak, B., Katsoulidis, E., Carayol, N., Altman, J.K., Redig, A.J., Tallman, M.S., 844 Ueda, T., Watanabe-Fukunaga, R., Fukunaga, R., and Platanias, L.C. (2008). 845 Regulation of arsenic trioxide-induced cellular responses by Mnk1 and Mnk2. J Biol 846 Chem 283, 12034-12042. 
847 Elizalde, P.V., Cordo Russo, R.I., Chervo, M.F., and Schillaci, R. (2016). ErbB-2 848 nuclear function in breast cancer growth, metastasis and resistance to therapy. Endocr 849 Relat Cancer 23, T243-T257.

850 Eto, I. (2010). Upstream molecular signaling pathways of p27(Kip1) expression: effects 851 of 4-hydroxytamoxifen, dexamethasone, and retinoic acids. Cancer Cell Int 10, 3.

852 Faivre, E., Skildum, A., Pierson-Mullany, L., and Lange, C.A. (2005). Integration of 853 progesterone receptor mediated rapid signaling and nuclear actions in breast cancer cell 854 models: role of mitogen-activated protein kinases and cell cycle regulators. Steroids 70, $855 \quad 418-426$.

856 Fu, X.D., Goglia, L., Sanchez, A.M., Flamini, M., Giretti, M.S., Tosi, V., Genazzani, 857 A.R., and Simoncini, T. (2010). Progesterone receptor enhances breast cancer cell 858 motility and invasion via extranuclear activation of focal adhesion kinase. Endocr Relat 859 Cancer 17, 431-443.

860 Garg, D., Ng, S.S.M., Baig, K.M., Driggers, P., and Segars, J. (2017). Progesterone861 Mediated Non-Classical Signaling. Trends Endocrinol Metab 28, 656-668.

862 Gonzalez-Suarez, E., Jacob, A.P., Jones, J., Miller, R., Roudier-Meyer, M.P., Erwert, 863 R., Pinkas, J., Branstetter, D., and Dougall, W.C. (2010). RANK ligand mediates 864 progestin-induced mammary epithelial proliferation and carcinogenesis. Nature 468, $865 \quad 103-107$.

866 Grimmler, M., Wang, Y., Mund, T., Cilensek, Z., Keidel, E.M., Waddell, M.B., Jakel, 867 H., Kullmann, M., Kriwacki, R.W., and Hengst, L. (2007). Cdk-inhibitory activity and 
868

869

870

871

872

873

875

876

877

878

879

880

881

882

883

884

885

886

887

stability of p27Kip1 are directly regulated by oncogenic tyrosine kinases. Cell 128 , 269-280.

Guo, Q., Li, V.Z., Nichol, J.N., Huang, F., Yang, W., Preston, S.E.J., Talat, Z., Lefrere, H., Yu, H., Zhang, G., et al. (2019). MNK1/NODAL Signaling Promotes Invasive Progression of Breast Ductal Carcinoma In Situ. Cancer Res 79, 1646-1657.

Gyorffy, B., Lanczky, A., Eklund, A.C., Denkert, C., Budczies, J., Li, Q., and Szallasi, Z. (2010). An online survival analysis tool to rapidly assess the effect of 22,277 genes on breast cancer prognosis using microarray data of 1,809 patients. Breast Cancer Res Treat $123,725-731$.

Hadad, S.M., Fleming, S., and Thompson, A.M. (2008). Targeting AMPK: a new therapeutic opportunity in breast cancer. Crit Rev Oncol Hematol 67, 1-7.

Hagan, C.R., Knutson, T.P., and Lange, C.A. (2013). A Common Docking Domain in Progesterone Receptor-B links DUSP6 and CK2 signaling to proliferative transcriptional programs in breast cancer cells. Nucleic Acids Res 41, 8926-8942.

Hassa, P.O., Haenni, S.S., Elser, M., and Hottiger, M.O. (2006). Nuclear ADPribosylation reactions in mammalian cells: where are we today and where are we going? Microbiol Mol Biol Rev 70, 789-829.

Hatzoglou, E., and Sekeris, C.E. (1997). The detection of nucleotide sequences with strong similarity to hormone responsive elements in the genome of eubacteria and archaebacteria and their possible relation to similar sequences present in the mitochondrial genome. J Theor Biol 184, 339-344. 
889 Haverinen, M., Passinen, S., Syvala, H., Pasanen, S., Manninen, T., Tuohimaa, P., and

890 Ylikomi, T. (2001). Heat shock protein 90 and the nuclear transport of progesterone 891 receptor. Cell Stress Chaperones 6, 256-262.

892 Hiratani, K., Haruta, T., Tani, A., Kawahara, J., Usui, I., and Kobayashi, M. (2005). 893 Roles of mTOR and JNK in serine phosphorylation, translocation, and degradation of 894 IRS-1. Biochem Biophys Res Commun 335, 836-842.

895 Hoch, N.C., and Polo, L.M. (2019). ADP-ribosylation: from molecular mechanisms to 896 human disease. Genet Mol Biol 43, e20190075.

897 Holaska, J.M., and Wilson, K.L. (2007). An emerin "proteome": purification of distinct 898 emerin-containing complexes from HeLa cells suggests molecular basis for diverse 899 roles including gene regulation, mRNA splicing, signaling, mechanosensing, and 900 nuclear architecture. Biochemistry 46, 8897-8908.

901 Hsu, J.L., and Hung, M.C. (2016). The role of HER2, EGFR, and other receptor 902 tyrosine kinases in breast cancer. Cancer Metastasis Rev 35, 575-588.

903 Huang, E.J., and Reichardt, L.F. (2003). Trk receptors: roles in neuronal signal 904 transduction. Annu Rev Biochem 72, 609-642.

905 Hurley, R.L., Anderson, K.A., Franzone, J.M., Kemp, B.E., Means, A.R., and Witters, 906 L.A. (2005). The Ca2+/calmodulin-dependent protein kinase kinases are AMP907 activated protein kinase kinases. J Biol Chem 280, 29060-29066.

908 Ibrahim, Y.H., Byron, S.A., Cui, X., Lee, A.V., and Yee, D. (2008). Progesterone 909 receptor-B regulation of insulin-like growth factor-stimulated cell migration in breast 910 cancer cells via insulin receptor substrate-2. Mol Cancer Res 6, 1491-1498. 
911 Jin, W. (2020). Roles of TrkC Signaling in the Regulation of Tumorigenicity and

912 Metastasis of Cancer. Cancers (Basel) 12.

913 Kato, S., Endoh, H., Masuhiro, Y., Kitamoto, T., Uchiyama, S., Sasaki, H., Masushige,

914 S., Gotoh, Y., Nishida, E., Kawashima, H., et al. (1995). Activation of the estrogen

915 receptor through phosphorylation by mitogen-activated protein kinase. Science 270 , 916 1491-1494.

917 Knotts, T.A., Orkiszewski, R.S., Cook, R.G., Edwards, D.P., and Weigel, N.L. (2001).

918 Identification of a phosphorylation site in the hinge region of the human progesterone 919 receptor and additional amino-terminal phosphorylation sites. J Biol Chem 276, 84759208483.

921 Knutson, T.P., Truong, T.H., Ma, S., Brady, N.J., Sullivan, M.E., Raj, G., Schwertfeger, 922 K.L., and Lange, C.A. (2017). Posttranslationally modified progesterone receptors 923 direct ligand-specific expression of breast cancer stem cell-associated gene programs. 924 J Hematol Oncol 10, 89.

925 Kramer, E.A., Seeger, H., Kramer, B., Wallwiener, D., and Mueck, A.O. (2006). 926 Characterization of the stimulatory effect of medroxyprogesterone acetate and 927 chlormadinone acetate on growth factor treated normal human breast epithelial cells. J 928 Steroid Biochem Mol Biol 98, 174-178.

929 Lange, C.A. (2008). Integration of progesterone receptor action with rapid signaling 930 events in breast cancer models. J Steroid Biochem Mol Biol 108, 203-212. 
931 Lange, C.A., Shen, T., and Horwitz, K.B. (2000). Phosphorylation of human 932 progesterone receptors at serine-294 by mitogen-activated protein kinase signals their 933 degradation by the 26S proteasome. Proc Natl Acad Sci U S A 97, 1032-1037.

934 Le Dily, F., Vidal, E., Cuartero, Y., Quilez, J., Nacht, A.S., Vicent, G.P., Carbonell935 Caballero, J., Sharma, P., Villanueva-Canas, J.L., Ferrari, R., et al. (2019). Hormone936 control regions mediate steroid receptor-dependent genome organization. Genome Res 937 29, 29-39.

938 Lee, II, and Kim, J.J. (2014). Influence of AKT on progesterone action in endometrial 939 diseases. Biol Reprod 91, 63.

940 Leidecker, O., Bonfiglio, J.J., Colby, T., Zhang, Q., Atanassov, I., Zaja, R., Palazzo, 941 L., Stockum, A., Ahel, I., and Matic, I. (2016). Serine is a new target residue for 942 endogenous ADP-ribosylation on histones. Nat Chem Biol 12, 998-1000.

943 Leung, A.K. (2014). Poly(ADP-ribose): an organizer of cellular architecture. J Cell Biol $944205,613-619$.

945 Li, X., Wong, J., Tsai, S.Y., Tsai, M.J., and O'Malley, B.W. (2003). Progesterone and 946 glucocorticoid receptors recruit distinct coactivator complexes and promote distinct 947 patterns of local chromatin modification. Mol Cell Biol 23, 3763-3773.

948 Liberzon, A., Subramanian, A., Pinchback, R., Thorvaldsdottir, H., Tamayo, P., and 949 Mesirov, J.P. (2011). Molecular signatures database (MSigDB) 3.0. Bioinformatics 27, $950 \quad 1739-1740$.

951 Liu, Q., Florea, B.I., and Filippov, D.V. (2017). ADP-Ribosylation Goes Normal: 952 Serine as the Major Site of the Modification. Cell Chem Biol 24, 431-432. 
953 Malbeteau, L., Poulard, C., Languilaire, C., Mikaelian, I., Flamant, F., Le Romancer,

954 M., and Corbo, L. (2020). PRMT1 Is Critical for the Transcriptional Activity and the 955 Stability of the Progesterone Receptor. iScience 23, 101236.

956 Mamay, C.L., Mingo-Sion, A.M., Wolf, D.M., Molina, M.D., and Van Den Berg, C.L. 957 (2003). An inhibitory function for JNK in the regulation of IGF-I signaling in breast 958 cancer. Oncogene 22, 602-614.

959 Martello, R., Leutert, M., Jungmichel, S., Bilan, V., Larsen, S.C., Young, C., Hottiger, 960 M.O., and Nielsen, M.L. (2016). Proteome-wide identification of the endogenous ADP961 ribosylome of mammalian cells and tissue. Nat Commun 7, 12917.

962 McInnes, K.J., Brown, K.A., Hunger, N.I., and Simpson, E.R. (2012). Regulation of 963 LKB1 expression by sex hormones in adipocytes. Int J Obes (Lond) 36, 982-985.

964 McInnes, K.J., Corbould, A., Simpson, E.R., and Jones, M.E. (2006). Regulation of 965 adenosine 5',monophosphate-activated protein kinase and lipogenesis by androgens 966 contributes to visceral obesity in an estrogen-deficient state. Endocrinology 147, 59079675913.

968 McKay, M.M., Ritt, D.A., and Morrison, D.K. (2009). Signaling dynamics of the KSR1 969 scaffold complex. Proc Natl Acad Sci U S A 106, 11022-11027.

970 Meng, L., Liu, B., Ji, R., Jiang, X., Yan, X., and Xin, Y. (2019). Targeting the $971 \mathrm{BDNF} / \mathrm{TrkB}$ pathway for the treatment of tumors. Oncol Lett 17, 2031-2039.

972 Messner, S., Altmeyer, M., Zhao, H., Pozivil, A., Roschitzki, B., Gehrig, P., 973 Rutishauser, D., Huang, D., Caflisch, A., and Hottiger, M.O. (2010). PARP1 ADP974 ribosylates lysine residues of the core histone tails. Nucleic Acids Res 38, 6350-6362. 
975 Migliaccio, A., Piccolo, D., Castoria, G., Di Domenico, M., Bilancio, A., Lombardi, 976 M., Gong, W., Beato, M., and Auricchio, F. (1998). Activation of the Src/p21ras/Erk 977 pathway by progesterone receptor via cross-talk with estrogen receptor. EMBO J 17, $978 \quad 2008-2018$.

979 Montero, J.C., Esparis-Ogando, A., Re-Louhau, M.F., Seoane, S., Abad, M., Calero, 980 R., Ocana, A., and Pandiella, A. (2014). Active kinase profiling, genetic and 981 pharmacological data define mTOR as an important common target in triple-negative 982 breast cancer. Oncogene 33, 148-156.

983 Nacht, A.S., Pohl, A., Zaurin, R., Soronellas, D., Quilez, J., Sharma, P., Wright, R.H., 984 Beato, M., and Vicent, G.P. (2016). Hormone-induced repression of genes requires 985 BRG1-mediated H1.2 deposition at target promoters. EMBO J 35, 1822-1843.

986 Nelson, M.B., Nyhus, J.K., Oravecz-Wilson, K.I., and Barbera-Guillem, E. (2001). 987 Tumor cells express FcgammaRI which contributes to tumor cell growth and a 988 metastatic phenotype. Neoplasia 3, 115-124.

989 Ortega, M.A., Fraile-Martinez, O., Asunsolo, A., Bujan, J., Garcia-Honduvilla, N., and 990 Coca, S. (2020). Signal Transduction Pathways in Breast Cancer: The Important Role 991 of PI3K/Akt/mTOR. J Oncol 2020, 9258396.

992 Park, J., Wood, M.A., and Cole, M.D. (2002). BAF53 forms distinct nuclear complexes 993 and functions as a critical c-Myc-interacting nuclear cofactor for oncogenic 994 transformation. Mol Cell Biol 22, 1307-1316. 
995 Paul, A., Edwards, J., Pepper, C., and Mackay, S. (2018). Inhibitory-kappaB Kinase 996 (IKK) alpha and Nuclear Factor-kappaB (NFkappaB)-Inducing Kinase (NIK) as Anti997 Cancer Drug Targets. Cells 7.

998 Pedram, A., Razandi, M., Sainson, R.C., Kim, J.K., Hughes, C.C., and Levin, E.R. 999 (2007). A conserved mechanism for steroid receptor translocation to the plasma 1000 membrane. J Biol Chem 282, 22278-22288.

1001 Pennington, K.L., Chan, T.Y., Torres, M.P., and Andersen, J.L. (2018). The dynamic 1002 and stress-adaptive signaling hub of 14-3-3: emerging mechanisms of regulation and 1003 context-dependent protein-protein interactions. Oncogene 37, 5587-5604.

1004 Pickup, K.E., Pardow, F., Carbonell-Caballero, J., Lioutas, A., Villanueva-Canas, J.L., 1005 Wright, R.H.G., and Beato, M. (2019). Expression of Oncogenic Drivers in 3D Cell 1006 Culture Depends on Nuclear ATP Synthesis by NUDT5. Cancers (Basel) 11 .

1007 Pina, B., Bruggemeier, U., and Beato, M. (1990). Nucleosome positioning modulates 1008 accessibility of regulatory proteins to the mouse mammary tumor virus promoter. Cell $100960,719-731$.

1010 Price, T.M., and Dai, Q. (2015). The Role of a Mitochondrial Progesterone Receptor 1011 (PR-M) in Progesterone Action. Semin Reprod Med 33, 185-194.

1012 Proietti, C.J., Rosemblit, C., Beguelin, W., Rivas, M.A., Diaz Flaque, M.C., Charreau, 1013 E.H., Schillaci, R., and Elizalde, P.V. (2009). Activation of Stat3 by heregulin/ErbB-2 1014 through the co-option of progesterone receptor signaling drives breast cancer growth. 1015 Mol Cell Biol 29, 1249-1265. 
1016 Qiu, M., Olsen, A., Faivre, E., Horwitz, K.B., and Lange, C.A. (2003). Mitogen-

1017 activated protein kinase regulates nuclear association of human progesterone receptors.

1018 Mol Endocrinol 17, 628-642.

1019 Ranade, D., Pradhan, R., Jayakrishnan, M., Hegde, S., and Sengupta, K. (2019). Lamin

$1020 \mathrm{~A} / \mathrm{C}$ and Emerin depletion impacts chromatin organization and dynamics in the 1021 interphase nucleus. BMC Mol Cell Biol 20, 11.

1022 Regua, A.T., Doheny, D., Arrigo, A., and Lo, H.W. (2019). Trk receptor tyrosine 1023 kinases in metastasis and cancer therapy. Discov Med 28, 195-203.

1024 Reichardt, L.F. (2006). Neurotrophin-regulated signalling pathways. Philos Trans R 1025 Soc Lond B Biol Sci 361, 1545-1564.

1026 Rider, V., Isuzugawa, K., Twarog, M., Jones, S., Cameron, B., Imakawa, K., and Fang,

1027 J. (2006). Progesterone initiates Wnt-beta-catenin signaling but estradiol is required for 1028 nuclear activation and synchronous proliferation of rat uterine stromal cells. $\mathrm{J}$ 1029 Endocrinol 191, 537-548.

1030 Sanchez-Margalet, V., and Najib, S. (2001). Sam68 is a docking protein linking GAP 1031 and PI3K in insulin receptor signaling. Mol Cell Endocrinol 183, 113-121.

1032 Schramek, D., Leibbrandt, A., Sigl, V., Kenner, L., Pospisilik, J.A., Lee, H.J., Hanada, 1033 R., Joshi, P.A., Aliprantis, A., Glimcher, L., et al. (2010). Osteoclast differentiation 1034 factor RANKL controls development of progestin-driven mammary cancer. Nature $1035468,98-102$. 
Shannon, P., Markiel, A., Ozier, O., Baliga, N.S., Wang, J.T., Ramage, D., Amin, N.,

1037 Schwikowski, B., and Ideker, T. (2003). Cytoscape: a software environment for

1038 integrated models of biomolecular interaction networks. Genome Res 13, 2498-2504.

1039 Shostak, K., and Chariot, A. (2011). NF-kappaB, stem cells and breast cancer: the links 1040 get stronger. Breast Cancer Res 13, 214.

1041 Skildum, A., Faivre, E., and Lange, C.A. (2005). Progesterone receptors induce cell 1042 cycle progression via activation of mitogen-activated protein kinases. Mol Endocrinol $104319,327-339$.

1044 Smith, D.F. (1993). Dynamics of heat shock protein 90-progesterone receptor binding 1045 and the disactivation loop model for steroid receptor complexes. Mol Endocrinol 7, 1046 1418-1429.

1047 Subramanian, A., Tamayo, P., Mootha, V.K., Mukherjee, S., Ebert, B.L., Gillette, 1048 M.A., Paulovich, A., Pomeroy, S.L., Golub, T.R., Lander, E.S., et al. (2005). Gene set 1049 enrichment analysis: a knowledge-based approach for interpreting genome-wide 1050 expression profiles. Proc Natl Acad Sci U S A 102, 15545-15550.

1051 Takahashi, A., Kato, K., Kuboyama, A., Inoue, T., Tanaka, Y., Kuhara, A., Kinoshita, 1052 K., Takeda, S., and Wake, N. (2009). Induction of senescence by progesterone receptor1053 B activation in response to cAMP in ovarian cancer cells. Gynecol Oncol 113, 270-276.

1054 Thomas, C., and Tulin, A.V. (2013). Poly-ADP-ribose polymerase: machinery for 1055 nuclear processes. Mol Aspects Med 34, 1124-1137. 
1056

1057

1058

1059

1060

1061

1062

1063

1064

1065

1066

1067

1068

1069

1070

1071

1072

1073

1074

1075

1076

1077

Trevino, L.S., Bolt, M.J., Grimm, S.L., Edwards, D.P., Mancini, M.A., and Weigel, N.L. (2016). Differential Regulation of Progesterone Receptor-Mediated Transcription by CDK2 and DNA-PK. Mol Endocrinol 30, 158-172.

Tzatsos, A. (2009). Raptor binds the SAIN (Shc and IRS-1 NPXY binding) domain of insulin receptor substrate-1 (IRS-1) and regulates the phosphorylation of IRS-1 at Ser636/639 by mTOR. J Biol Chem 284, 22525-22534.

Tzivion, G., Dobson, M., and Ramakrishnan, G. (2011). FoxO transcription factors; Regulation by AKT and 14-3-3 proteins. Biochim Biophys Acta 1813, 1938-1945.

Tzivion, G., and Hay, N. (2011). PI3K-AKT-FoxO axis in cancer and aging. Biochim Biophys Acta 1813, 1925.

Uhlen, M., Fagerberg, L., Hallstrom, B.M., Lindskog, C., Oksvold, P., Mardinoglu, A., Sivertsson, A., Kampf, C., Sjostedt, E., Asplund, A., et al. (2015). Proteomics. Tissuebased map of the human proteome. Science 347, 1260419.

Valverde, A.M., Arribas, M., Mur, C., Navarro, P., Pons, S., Cassard-Doulcier, A.M., Kahn, C.R., and Benito, M. (2003). Insulin-induced up-regulated uncoupling protein-1 expression is mediated by insulin receptor substrate 1 through the phosphatidylinositol 3-kinase/Akt signaling pathway in fetal brown adipocytes. J Biol Chem 278, 1022110231.

Vicent, G.P., Ballare, C., Nacht, A.S., Clausell, J., Subtil-Rodriguez, A., Quiles, I., Jordan, A., and Beato, M. (2006). Induction of progesterone target genes requires activation of Erk and Msk kinases and phosphorylation of histone H3. Mol Cell 24, $367-381$ 
Vicent, G.P., Nacht, A.S., Font-Mateu, J., Castellano, G., Gaveglia, L., Ballare, C., and Beato, M. (2011). Four enzymes cooperate to displace histone H1 during the first minute of hormonal gene activation. Genes Dev 25, 845-862.

Vicent, G.P., Wright, R.H., and Beato, M. (2016). Linker histones in hormonal gene regulation. Biochim Biophys Acta 1859, 520-525.

Vicent, G.P., Zaurin, R., Ballare, C., Nacht, A.S., and Beato, M. (2009a). Erk signaling and chromatin remodeling in MMTV promoter activation by progestins. Nucl Recept Signal 7, e008.

Vicent, G.P., Zaurin, R., Nacht, A.S., Font-Mateu, J., Le Dily, F., and Beato, M. (2010). Nuclear factor 1 synergizes with progesterone receptor on the mouse mammary tumor virus promoter wrapped around a histone $\mathrm{H} 3 / \mathrm{H} 4$ tetramer by facilitating access to the central hormone-responsive elements. J Biol Chem 285, 2622-2631.

Vicent, G.P., Zaurin, R., Nacht, A.S., Li, A., Font-Mateu, J., Le Dily, F., Vermeulen, M., Mann, M., and Beato, M. (2009b). Two chromatin remodeling activities cooperate during activation of hormone responsive promoters. PLoS Genet 5, e1000567.

von Eyss, B., Jaenicke, L.A., Kortlever, R.M., Royla, N., Wiese, K.E., Letschert, S., McDuffus, L.A., Sauer, M., Rosenwald, A., Evan, G.I., et al. (2015). A MYC-Driven Change in Mitochondrial Dynamics Limits YAP/TAZ Function in Mammary Epithelial Cells and Breast Cancer. Cancer Cell 28, 743-757.

Wang, R., Fu, L., Li, J., Zhao, D., Zhao, Y., and Yin, L. (2020). Microarray Analysis for Differentially Expressed Genes Between Stromal and Epithelial Cells in Development and Metastasis of Invasive Breast Cancer. J Comput Biol. 
1100 Wang, Y., Feng, H., Bi, C., Zhu, L., Pollard, J.W., and Chen, B. (2007). GSK-3beta

1101 mediates in the progesterone inhibition of estrogen induced cyclin D2 nuclear

1102 localization and cell proliferation in cyclin D1-/- mouse uterine epithelium. FEBS Lett $1103581,3069-3075$.

1104 Warde-Farley, D., Donaldson, S.L., Comes, O., Zuberi, K., Badrawi, R., Chao, P.,

1105 Franz, M., Grouios, C., Kazi, F., Lopes, C.T., et al. (2010). The GeneMANIA 1106 prediction server: biological network integration for gene prioritization and predicting 1107 gene function. Nucleic Acids Res 38, W214-220.

1108 Wheater, M.J., Johnson, P.W., and Blaydes, J.P. (2010). The role of MNK proteins and 1109 eIF4E phosphorylation in breast cancer cell proliferation and survival. Cancer Biol Ther $111010,728-735$.

1111 Wierer, M., Verde, G., Pisano, P., Molina, H., Font-Mateu, J., Di Croce, L., and Beato, 1112 M. (2013). PLK1 signaling in breast cancer cells cooperates with estrogen receptor1113 dependent gene transcription. Cell Rep 3, 2021-2032.

1114 Wright, R.H., Castellano, G., Bonet, J., Le Dily, F., Font-Mateu, J., Ballare, C., Nacht, 1115 A.S., Soronellas, D., Oliva, B., and Beato, M. (2012). CDK2-dependent activation of 1116 PARP-1 is required for hormonal gene regulation in breast cancer cells. Genes Dev 26, 1117 1972-1983.

1118 Wright, R.H., Lioutas, A., Le Dily, F., Soronellas, D., Pohl, A., Bonet, J., Nacht, A.S., 1119 Samino, S., Font-Mateu, J., Vicent, G.P., et al. (2016). ADP-ribose-derived nuclear 1120 ATP synthesis by NUDIX5 is required for chromatin remodeling. Science 352, 1221$1121 \quad 1225$. 
1122 Wright, R.H.G., Le Dily, F., and Beato, M. (2019). ATP, $\operatorname{Mg}(2+)$, Nuclear Phase

1123 Separation, and Genome Accessibility. Trends Biochem Sci 44, 565-574.

1124 Wu, L., Huang, X.J., Yang, C.H., Deng, S.S., Qian, M., Zang, Y., and Li, J. (2011). 5'-

1125 AMP-activated protein kinase (AMPK) regulates progesterone receptor transcriptional

1126 activity in breast cancer cells. Biochem Biophys Res Commun 416, 172-177.

1127 Xu, B., Bird, V.G., and Miller, W.T. (1995). Substrate specificities of the insulin and

1128 insulin-like growth factor 1 receptor tyrosine kinase catalytic domains. J Biol Chem $1129270,29825-29830$.

1130 Yamaguchi, H., and Taouk, G.M. (2020). A Potential Role of YAP/TAZ in the

1131 Interplay Between Metastasis and Metabolic Alterations. Front Oncol 10, 928.

1132 Zhao, H., Orhan, Y.C., Zha, X., Esencan, E., Chatterton, R.T., and Bulun, S.E. (2017).

1133 AMP-activated protein kinase and energy balance in breast cancer. Am J Transl Res 9, 1134 197-213.

1135

1136 Figure Legends

1137

1138 Fig. 1. Targeted Antibody Array Phosphorylation data following hormone.

1140 A) Schematic overview of experimental procedure. Synchronised T47D cells were

1141 exposed to hormone for the length of time indicated. Triplicate samples were harvested

1142 and phosphorylated proteins were identified using an antibody microarray (see

1143 materials and methods). Data was $\log _{2}$ normalised resulting in a total of 246 significant

1144 phosphosites from 155 unique proteins. B) Number of regulated sites per time point, 
$1145 \log _{2} \mathrm{FC}$ (Fold change) $>0.6<-0.6$ versus time 0 . C) Breakdown of up $\left(>0.6 \log _{2} \mathrm{FC}\right)$ and 1146 down $\left(<-0.6 \log _{2}\right.$ FC) per time point versus T0. D) Functional classification of the 1147 proteins identified as significantly phosphorylated across all time points, individual

1148 time point functional analysis (per time point see Fig. S2C). E) Venn diagram showing 1149 the overlap of significantly regulated phosphorylation sites across all time points. F) $1150 \log _{2}$ FC following hormone of phosphorylated Mnk1 (T197/202) and EIF4E S209. G) 1151 Expression of EIF4E in normal versus breast tumour samples from breast cancer 1152 patients (Protein Atlas, see materials and methods). H) Kaplan Meyer overall survival 1153 stratifying patients based on the expression level of EIF4E in breast cancer data set 1154 ( $\mathrm{p}=5.3 \mathrm{e}-10)$. I) mRNA expression level of EIF4E in T47D cells treated with hormone. 1155 Dynamics of (J) p27/KIP T187 (K) CDK2 T160, ERK Y202/204. L). Expression level 1156 of total PR and CDK2 and phospho-CDK2 T160 in T47D breast cancer cells exposed 1157 to hormone in the presence or absence of ERK inhibitor (ERKii) as determined by 1158 western blotting using specific antibodies. (M) CDK2 T14/Y15 phosphorylation in 1159 response to hormone as determined by antibody array. Dynamics of (N) Cdc25C S216 1160 and (O) MAPKAPK2 T222 phosphorylation in response to hormone as determined by 1161 antibody array. P). Model for CDK2/ERK dynamic activation and deactivation in 1162 response to hormone based on the data presented in Fig. 1J-O.

1164 Fig. 2. Phosphosite enriched Shotgun Proteomics following hormone.

1166 A) T47D cells were treated with hormone at the times indicated. Biological triplicates 1167 were enriched for phosphopeptides using $\mathrm{TiO} 2$ followed by LC-MS-MS peptide 1168 identification. Data was $\log _{2}$ normalised resulting in a total of 310 phosphosites from 1169264 unique proteins. B) Volcano plots showing phosphopeptide $\log _{2} \mathrm{FC}$ versus p-value 
1170 for each of the time points following hormone. C) Number of significant phosphosites

1171 identified per time point. D) Analysis of the proportion of threonine, tyrosine and serine

1172 phosphorylated residues identified. E) Breakdown of up $\left(>0.6 \log _{2} \mathrm{FC}\right)$ and down $(<-0.6$

$1173 \log _{2}$ FC) per time point versus T0. F) Venn diagram showing the overlap of

1174 significantly regulated phosphorylation sites over time. G) Phosphorylation of

1175 progesterone receptor PR (S162), following progesterone validated by western blotting

1176 in presence or absence of CDK2 inhibitor $(\mathbf{H})$. Total PR levels are shown as a loading

1177 control.

1178

1179 Fig. 3. Combining Target Antibody Arrays and Shotgun Phosphoproteomic

1180 datasets following hormone

1181

1182 A) KEGG pathway enrichment analysis of proteins identified as regulated by

1183 phosphorylation in response to hormone. B) Cellular component analysis of

1184 phosphosites enriched per time point. Showing the hormone induced phosphorylation

1185 of the nucleoplasm and cytosol across all time points (group I) the activation of

1186 membrane raft proteins enriched at 1 minute (Group II) and phosphorylation of

1187 mitochondrial proteins enriched at 15 minutes (Group III), activation of nuclear

1188 structures; PML bodies and the nuclear matrix at 60 minutes (Group IV) and the

1189 activation of the cell-cell junctions and microtubules at 360 minutes (Group V). C) K

1190 mean clustering of all significantly regulated phosphorylation sites over time reveals 6

1191 distinct clusters. D) Similarity matrix of clusters 1-6 reveals similar dynamics for

1192 clusters 1 and 4 and an opposing similarity in phosphorylation dynamics for clusters 3

1193 and 6. Red indicates highly similar, well correlated, blue inversely correlated patterns

1194 of regulation. E) Word cloud showing the enrichment of GO-biological processes 
associated with proteins identified in similar clusters 1 and 4 "Early risers" which are regulated rapidly after hormone. F) Graph showing the opposing phosphorylation dynamic of proteins within clusters 3 and 6. G) Venn diagram showing the overlap of significantly identified Corum protein complexes identified in clusters 3 and 6 . H) Phosphorylation dynamic in response to hormone of Myc S373, and T58 and BAF53 S233. I) Expression level of BAF53 in tumour versus normal tissue within the TGCA dataset. J) Phosphorylation of AMPL T183 decreases rapidly in response to hormone. K) Model showing the key role of AMPK dephosphorylation in response to hormone in breast cancer cells, AMPK dephosphorylation is required in order for subsequent signaling cascades including NFkB, insulin, Hippo, JAK/STAT and mTOR to continue and the phosphorylation of PR S294 to take place.

\section{Fig. 4. Protein Complex analysis and Overlap of PARylation and Phosphorylation}

\section{in response to Progesterone.}

Heatmaps showing the phosphorylation of proteins within the Sam68-p85 IRS (A) and NF-kappa B (B) signalling complexes in response to hormone over time. C) Heatmap showing the phosphorylation of proteins of the p130 Cas-ER-Src-PI3K complex in response to hormone over time, the coordinated phosphorylation of each phosphosite individually is represented as a line graph (right panel). D) Venn diagram showing the overlap of proteins which contain either a phosphorylation site (379), PARylation site (1187) or both PTMs within the same protein after hormone exposure in breast cancer 
cells (52). E) Word cloud representation showing the GO-cellular component

1221 enrichment analysis of the 52 proteins identified as phosphorylated and PARylated in

1222 response to hormone (Fig. 4D). F) Heatmap showing the phosphorylation of

1223 components of the KRS1-RAF1-MEK signalling complex in response to hormone over

1224 time, all proteins shown are phosphorylated and PARylated and the dynamics of

1225 individual sites is shown on the right panel. G) Heatmap showing the phosphorylation

1226 proteins of the Emerin complex in response to hormone over time. H) Heatmap

1227 showing the phosphorylation of proteins (5/15) within the Kinase Maturation complex

1228 in response to hormone over time, phosphorylation dynamics of individual sites is

1229 shown (lower panel). I) Venn diagram showing the phosphorylation and or PARylation

1230 of proteins contained within the kinase maturation complex; 14/15 protein components

1231 of the complex contain at least one of the PTMs. J) Schematic representation of the

1232 complex components, PARylated proteins are indicated by blue circle, Phosphorylated

1233 by red (right panel).

1234

1235 Fig. 5. Combining PPI networks from distinct cellular compartments reveals a 1236 coordinated crosstalk.

1238 A) PPI network showing the significantly regulated phosphorylated proteins located in 1239 cell adhesion (blue) and the membrane raft (red) identified in response to hormone. B)

1240 Merge of the cell adhesion network (Fig. 5A, blue) and membrane raft network (Fig.

1241 5A red). The two networks connect based on known PPI however no protein was

1242 identified as annotated in both sets. This integration of the two networks is highlighted

1243 (right panel) where proteins from each network were selected based on having a first

1244 neighbour with a protein of the other network. C) Violin plot showing the average 
phosphorylation of proteins over time in response to hormone within the membrane raft or cell adhesion networks. Data is normalised to time $0=1$. D) Merge of Cell Adhesion-

1247 Membrane (Fig. 6B) and the cytoskeleton networks. The two networks are merged 1248 based on known PPI. Proteins annotated in more than one function are coloured based 1249 on the Venn diagram (i.e. cytoskeleton and cell Adhesion; light green, membrane raft 1250 and cytoskeleton; brown). E) Heatmap showing the average phosphorylation of all 1251 proteins within each network in response to hormone over time, showing the activation 1252 of the membrane raft first at 1 minute followed by the cytoskeleton and cell adhesion.

Fig. 6. Network Integration of signalling networks identified in response to

\section{5 hormone.}

A) PPI network showing the phosphorylated proteins present within the ERK signalling cascade (green) and the MAPK cascade (blue) identified in response to hormone. B)

1259 Merge of ERK-MAPK networks (Fig. 6A). The two networks are merged based on

1260 known PPI. Proteins annotated in both pathways are coloured based on the Venn 1261 diagram (fuchsia). C) PPI network showing the phosphorylated proteins present within 1262 the FC-receptor (yellow) and TRK-neurorophin (red) signalling pathways (yellow) 1263 identified in response to hormone (left and middle panel). D) Merge of FC-receptor and 1264 TRK neurotrophin networks. The two networks are merged based on known PPI. 1265 Proteins annotated in both pathways are coloured based on the Venn diagram (orange). 1266 E) Rapid and coordinated phosphorylation of FOXO1 S256 and FOXO S319 in 1267 response to hormone. Kaplan Meyer overall survival of patients stratified based on the 1268 expression of NTRK2 (F) and FOXO1 (G) in breast cancer patients ( $p=9.4 \mathrm{E}-10$ and 
6.8e-12 respectively). All networks, PPIs and integrated cascades are supplied in Cytoscape session 2.

\section{Supplementary Figure Legends}

1274

Fig. S1. Prior Knowledge Network (PKN) Progesterone Signalling.

A) Edge directed PKN network was manually curated from the literature. Annotated

1278 phosphorylation events, interactions, dissociations and cellular compartment are

1279 indicated. Network is available as a cytoscape network session (cys) or interaction (sys)

1280 file containing references for all edges present as shown in B) (See Supplementary File 1281 Network 1).

1282

1283 Fig. S2. Antibody Array controls and data analysis.

1284

1285 A) Schematic indicating the experimental procedure, quality control checks, and 1286 filtering applied to the antibody array experiments. B) Number of phosphosites 1287 identified per protein. Tau, PTK2, RPS6KA1 and RB1 are highlighted as they have 1288 multiple sites identified. C) Functional classification of the proteins identified as 1289 significantly phosphorylated at each time point D) KEGG pathway analysis, showing 1290 significant pathways (- $\log _{10}$ p-value) at each time point. E) Heatmap representation of 1291 GO biological process data, showing significant (- $\log _{10} \mathrm{p}$-value) processes at each time 1292 point. F) Heatmap representation of GO molecular function data, showing significant 1293 (- $\log _{10} \mathrm{p}$-value) functions at each time point. 
Fig. S3. Phosphoproteomic data acquisition and controls.

A) Correlation of triplicate samples from each of the time points. B) Number of

1298 phosphosites identified per protein, the proteins showing multiple sites per protein are

1299 highlighted. C) KEGG pathway analysis, showing significant pathways (- $\log _{10}$ p-

1300 value) at each time point. D) Heatmap representation of GO-biological process data,

1301 showing significant $\left(-\log _{10}\right.$ p-value) processes at each time point. E) Heatmap

1302 representation of GO-molecular function data, showing significant (- $\log _{10}$ p-value)

1303 functions per time point.

1304

Fig S4. Combining Antibody Array and Phosphoproteomic LC-MS-MS datasets.

A) Schematic representation showing the methodology and overlap combining

1308 antibody array and LC-MS-MS datasets. B) PCA analysis of phosphorylation datasets.

1309 C) Number of phosphosites identified per protein, the names of proteins showing 1310 multiple sites per protein are highlighted. D) Venn diagram showing the overlap of

1311 phosphosites per time point. E) Up and down regulated phosphorylation sites identified

1312 per time point. F) Phosphorylation levels of the proteins identified as significantly

1313 regulated after hormone located within the mitochondria. G) Analysis of the number of

1314 functions to which each unique protein was assigned $\mathbf{H}$ ) Venn diagram showing the 1315 overlap of protein functional class; Enzymes, Structural protein, Membrane-cell-cell 1316 contact, protein modulators and proteins with nucleic acid binding capacities.

1318 Fig S5. Functional Analysis of Proteins Identified. 
1320 All proteins were assigned one or more function based on GSEA database. Both Parent

1321 (outside/title), and children (within) are shown for each class and the proteins identified 1322 within that sub-group are shown. Nucleic acid binding (A), Membrane/Cell-cell contact 1323 (B), Protein Modulators (C), Enzymes (D), Cell signalling (E), and Structural proteins 1324 (F).

1326 Fig S6. Gene Ontology and Pathway analysis of combined dataset.

1328 A) Heatmap representation of GO biological process data, showing significant $\left(-\log _{10}\right.$ 1329 p-value) biological processes enrichment based on the protein phosphorylation at each 1330 time point. B) Heatmap representation of GO molecular function enrichment, showing 1331 significant (- $\log _{10} \mathrm{p}$-value) functions at each time point following hormone. C) KEGG 1332 pathway analysis, showing significant pathways (- $\log _{10} \mathrm{p}$-value) enriched at each time 1333 point following hormone exposure. D) Protein protein interaction (PPI) network 1334 generated using proteins identified as phosphorylated following hormone and were 1335 assigned as cytoskeleton located.

1337 Fig S7. Pathway Network Generation in Breast Cancer cells in response to 1338 Hormone.

1340 A) Protein protein interaction (PPI) network was generated using full phosphorylation 1341 dataset encompassing 321 proteins (Supplementary Material Network session 2) in 1342 Cytoscape using Genemania ${ }^{\mathrm{TM}}$ only considering protein-protein interactions with 1343 experimental evidence (Supp. Materials and methods), each node represents and 
1344 individual protein and interactions are represented by edges. Functional analysis was 1345 carried out to identify key pathways enriched within the full network (Full list

1346 Supplementary Table 16). Individual networks were generated from each function

1347 individually and are available within additional Network session 2. Graphs of several 1348 pathways determined to be enriched within the dataset are shown B) Fc receptor, C)

1349 MAPK, D) EGF E) ERK F) Insulin, G) TRK signalling, H) ERBB.

1351 Supplementary Table Legends

1353 Supplementary Table S1

1354

1355 Uniprot IDs of phosphorylated proteins identified in response to hormone. Time after 1356 hormone (minutes), data is normalized 0-1 row maximum and minimum.

1358 Supplementary Table S2

1359

1360 KEGG pathway enrichment; the pathway term, $p$ value and the proteins associated with 1361 the pathway are shown.

1363 Supplementary Table S3

1364

1365 Cellular component enrichment analysis of phosphorylated proteins. The time after 1366 hormone in which they peak, the adjusted $\mathrm{p}$ value, and proteins associated with each 1367 specific cellular component are given. 


\section{Supplementary Table S4}

1370

1371 Gene Ontology Biological Process enrichment analysis of phosphorylated proteins. The

1372 cluster in which the term is enriched, the adjusted $\mathrm{p}$ value, and proteins associated with 1373 each specific biological process are given.

1374

1375 Supplementary Table S5

1376

1377 Gene Ontology Molecular Function enrichment analysis of phosphorylated proteins.

1378 The cluster in which the term is enriched, the adjusted $\mathrm{p}$ value, and proteins associated

1379 with each specific molecular function are given.

1380

1381 Supplementary Table S6

1382

1383 Corum enrichment analysis of phosphorylated proteins. The p-value, and proteins 1384 associated with each complex are given.

1385

1386 Supplementary Table S7

1387

1388 Cellular component enrichment analysis of phosphorylated and PARylated proteins.

1389 The adjusted $\mathrm{p}$ value, and proteins associated with each specific cellular component are 1390 given.

1391

1392 Supplementary Table S8

1393 
1394 Corum enrichment analysis of phosphorylated and PARylated proteins. The p-value,

1395 and proteins associated with each complex are given, phosphorylated proteins are 1396 highlighted in yellow.

1398 Supplementary Table S9

1399

1400 Genemania analysis of phosphorylated proteins, all protein IDs are listed along with 1401 the GO: IDs for which they are associated.

1402

1403 Supplementary Table S10

1404

1405 Genemania analysis of phosphorylated proteins, the pathways enriched in Network 2

1406 are shown. The $\mathrm{q}$ value and the number of occurrences in the network versus the

1407 occurrences in the Network are shown.

1408

1409 Additional Files

1410

\section{Network Session 1}

1412

1413 Edge directed PKN network was manually curated from the literature. Annotated

1414 phosphorylation events, interactions, dissociations and cellular compartment are 1415 indicated.

1416

1417 Network Session 2 
1419 Protein protein interaction (PPI) network was generated using full phosphorylation

1420 dataset encompassing 321 proteins in Cytoscape using Genemania ${ }^{\mathrm{TM}}$ only considering

1421 protein-protein interactions with experimental evidence each node represents and

1422 individual protein and interactions are represented by edges. Functional analysis was

1423 carried out to identify key pathways enriched within the full network. Individual

1424 networks were generated from each function individually and are available as unique

1425 networks within Network session.

1426

1427 
Figures

Fig. 1

A

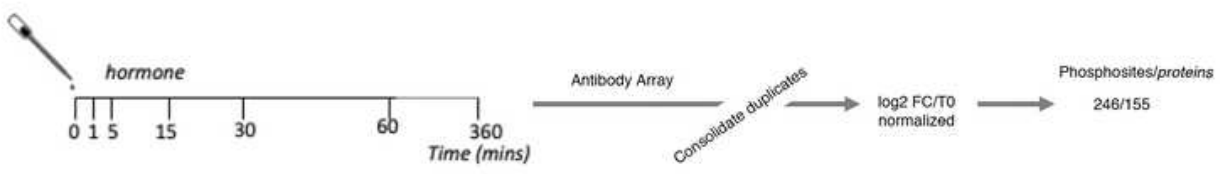

B

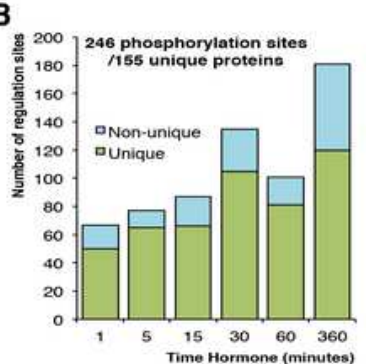

C
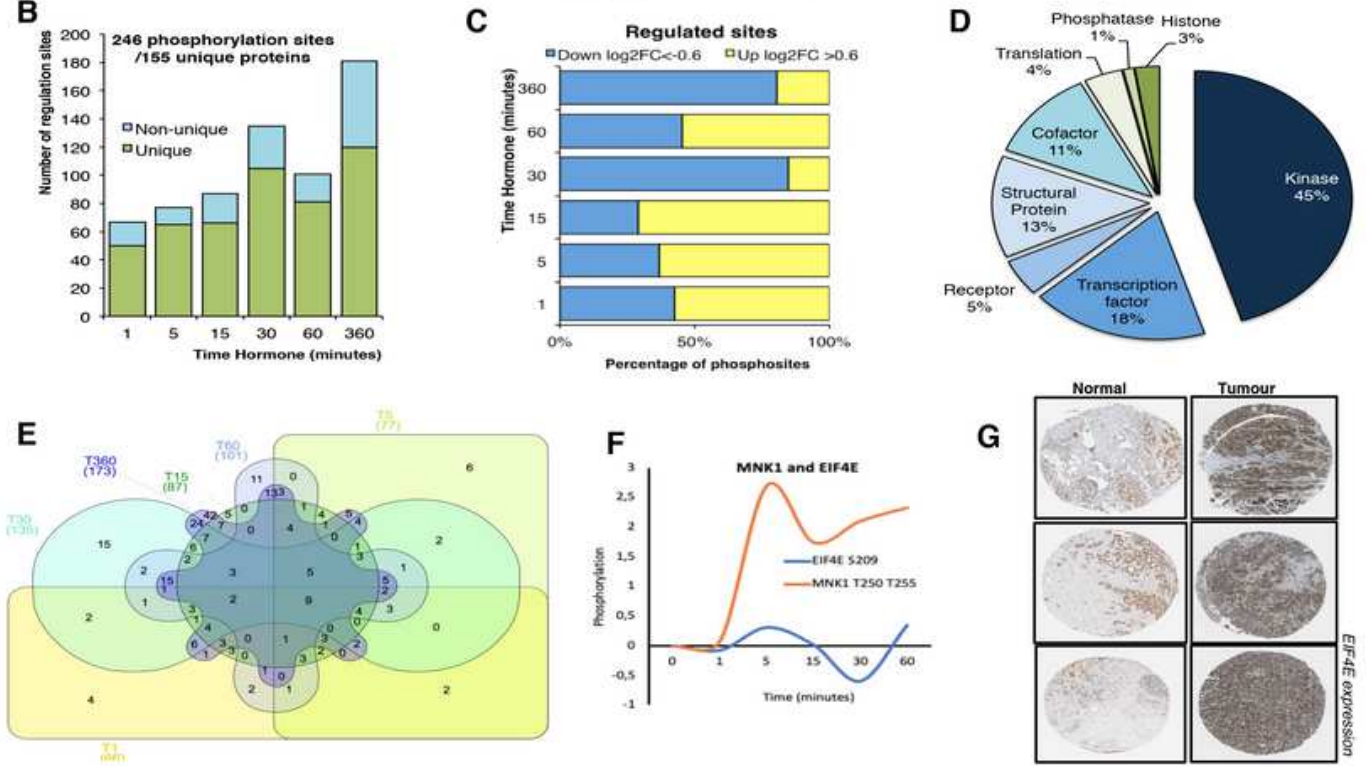

H
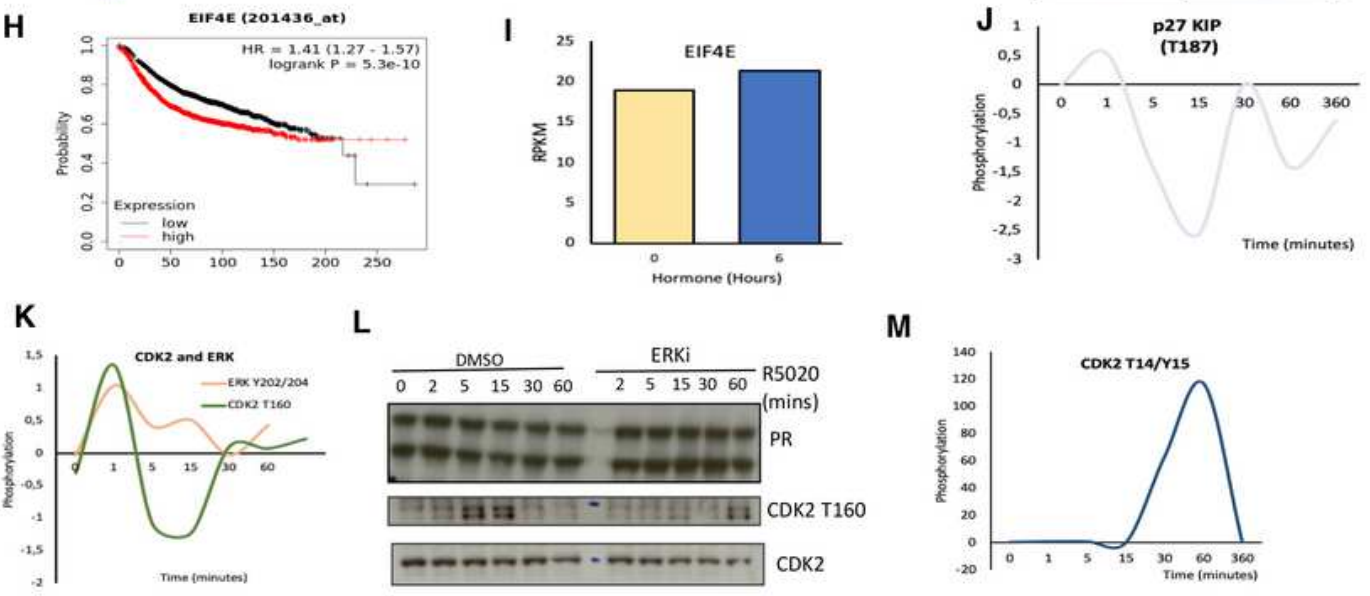

L

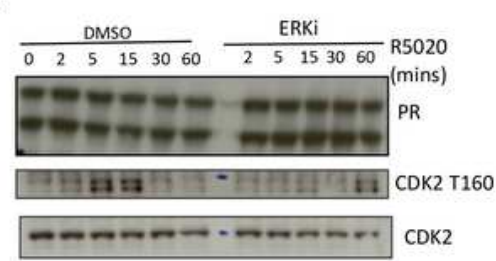

M
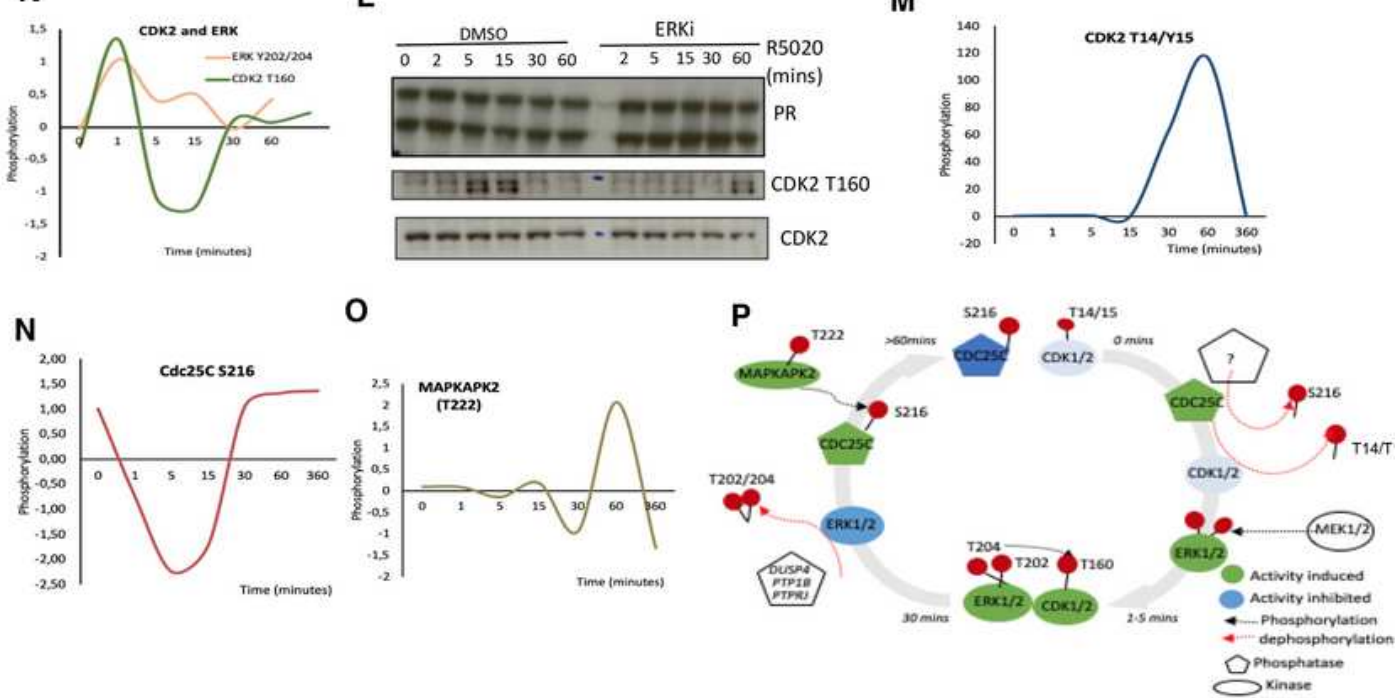

Figure 1

Targeted Antibody Array Phosphorylation data following hormone. A) Schematic overview of experimental procedure. Synchronised T47D cells were exposed to hormone for the length of time indicated. Triplicate samples were harvested and phosphorylated proteins were identified using an 
antibody microarray (see materials and methods). Data was log2 normalised resulting in a total of 246 significant phosphosites from 155 unique proteins. B) Number of regulated sites per time point, log2FC (Fold change) $>0.6<-0.6$ versus time 0 . C) Breakdown of up ( $>0.6 \log 2 \mathrm{FC}$ ) and down ( $<-0.6 \log 2 \mathrm{FC})$ per time point versus TO. D) Functional classification of the proteins identified as significantly phosphorylated across all time points, individual time point functional analysis (per time point see Fig. S2C). E) Venn diagram showing the overlap of significantly regulated phosphorylation sites across all time points. F) Log2 FC following hormone of phosphorylated Mnk1 (T197/202) and EIF4E S209. G) Expression of EIF4E in normal versus breast tumour samples from breast cancer patients (Protein Atlas, see materials and methods). $\mathrm{H}$ ) Kaplan Meyer overall survival stratifying patients based on the expression level of EIF4E in breast cancer data set $1154(p=5.3 e-10)$. I) mRNA expression level of EIF4E in T47D cells treated with hormone. Dynamics of (J) p27/KIP T187 (K) CDK2 T160, ERK Y202/204. L). Expression level 1156 of total PR and CDK2 and phospho-CDK2 T160 in T47D breast cancer cells exposed to hormone in the presence or absence of ERK inhibitor (ERKii) as determined by western blotting using specific antibodies. (M) CDK2 T14/Y15 phosphorylation in response to hormone as determined by antibody array. Dynamics of (N) Cdc25C S216 1160 and (O) MAPKAPK2 T222 phosphorylation in response to hormone as determined by 1161 antibody array. P). Model for CDK2/ERK dynamic activation and deactivation in response to hormone based on the data presented in Fig. $1 \mathrm{~J}-\mathrm{O}$ 
Fig. 2

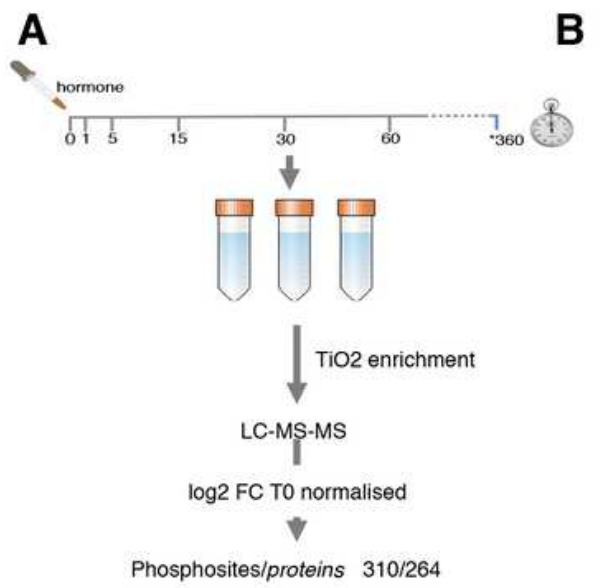

C

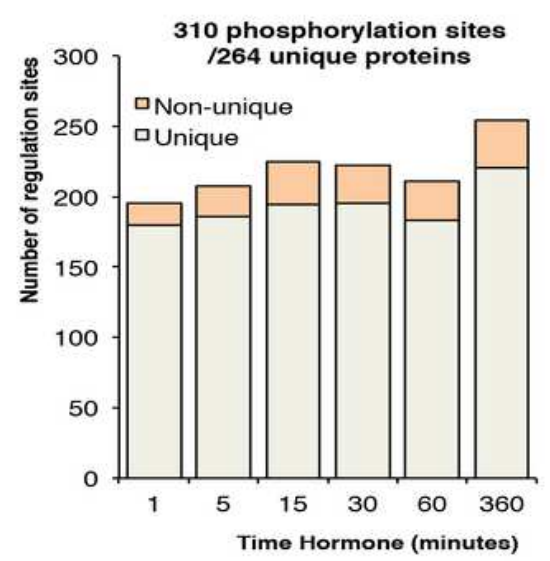

$\mathbf{F}$

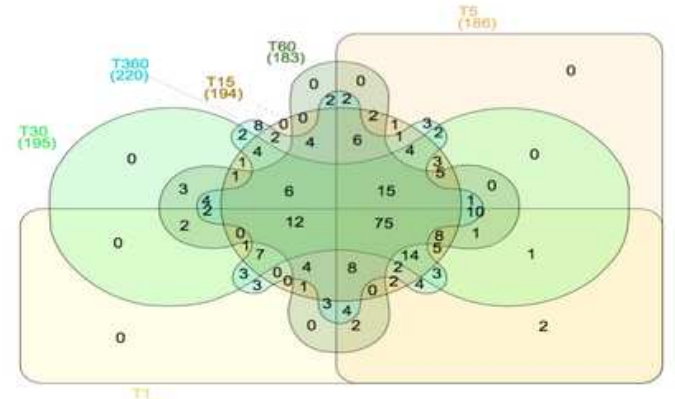

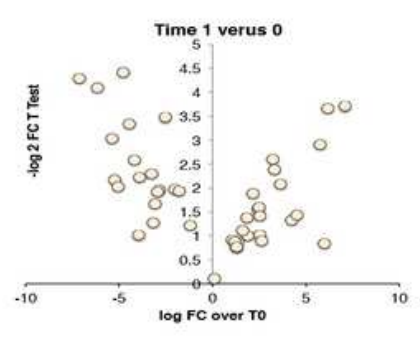
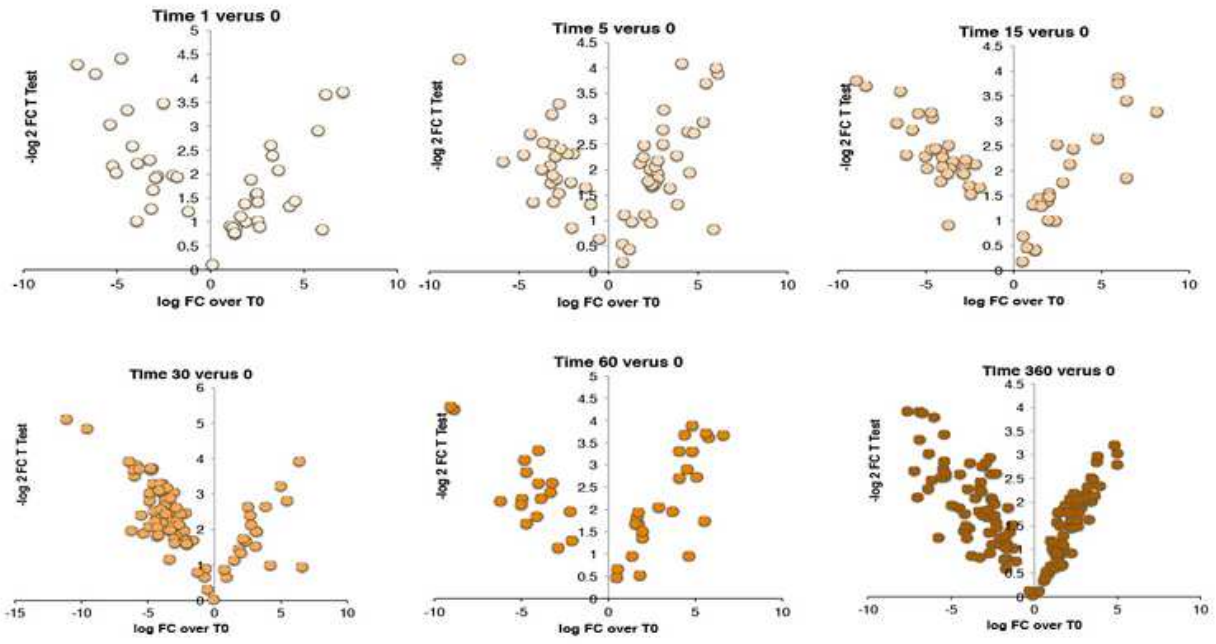

D

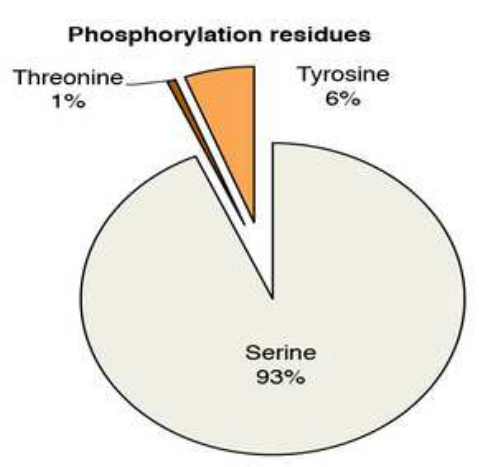

E

Regulated sites

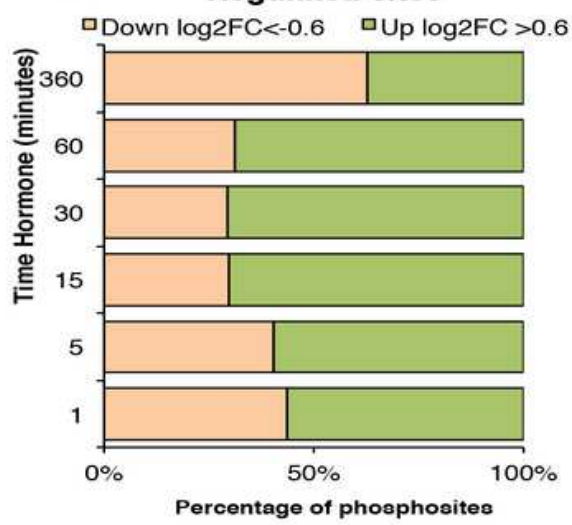

H

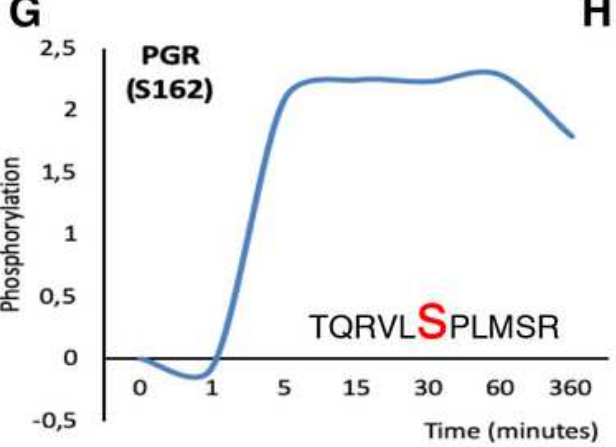

CDK2i - - - + + +

$\begin{array}{lllllll}P g & 0 & 5 & 30 & 0 & 5 & 30\end{array}$

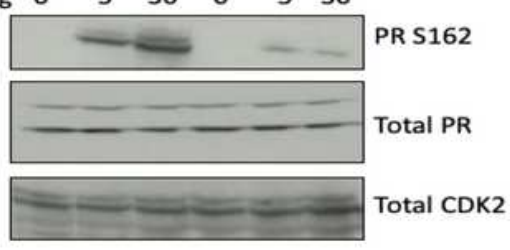

\section{Figure 2}

Phosphosite enriched Shotgun Proteomics following hormone. A) T47D cells were treated with hormone at the times indicated. Biological triplicates were enriched for phosphopeptides using TiO2 followed by LC-MS-MS peptide identification. Data was log2 normalised resulting in a total of 310 phosphosites from 264 unique proteins. B) Volcano plots showing phosphopeptide log2FC versus p-value for each of the time points following hormone. C) Number of significant phosphosites identified per time point. D) 
Analysis of the proportion of threonine, tyrosine and serine phosphorylated residues identified. E) Breakdown of up ( $>0.6 \log 2 \mathrm{FC}$ ) and down (<-0.6 $1173 \mathrm{log} 2 \mathrm{FC}$ ) per time point versus T0. F) Venn diagram showing the overlap of significantly regulated phosphorylation sites over time. G) Phosphorylation of progesterone receptor PR (S162), following progesterone validated by western blotting in presence or absence of CDK2 inhibitor (H). Total PR levels are shown as a loading control.

Fig. 3
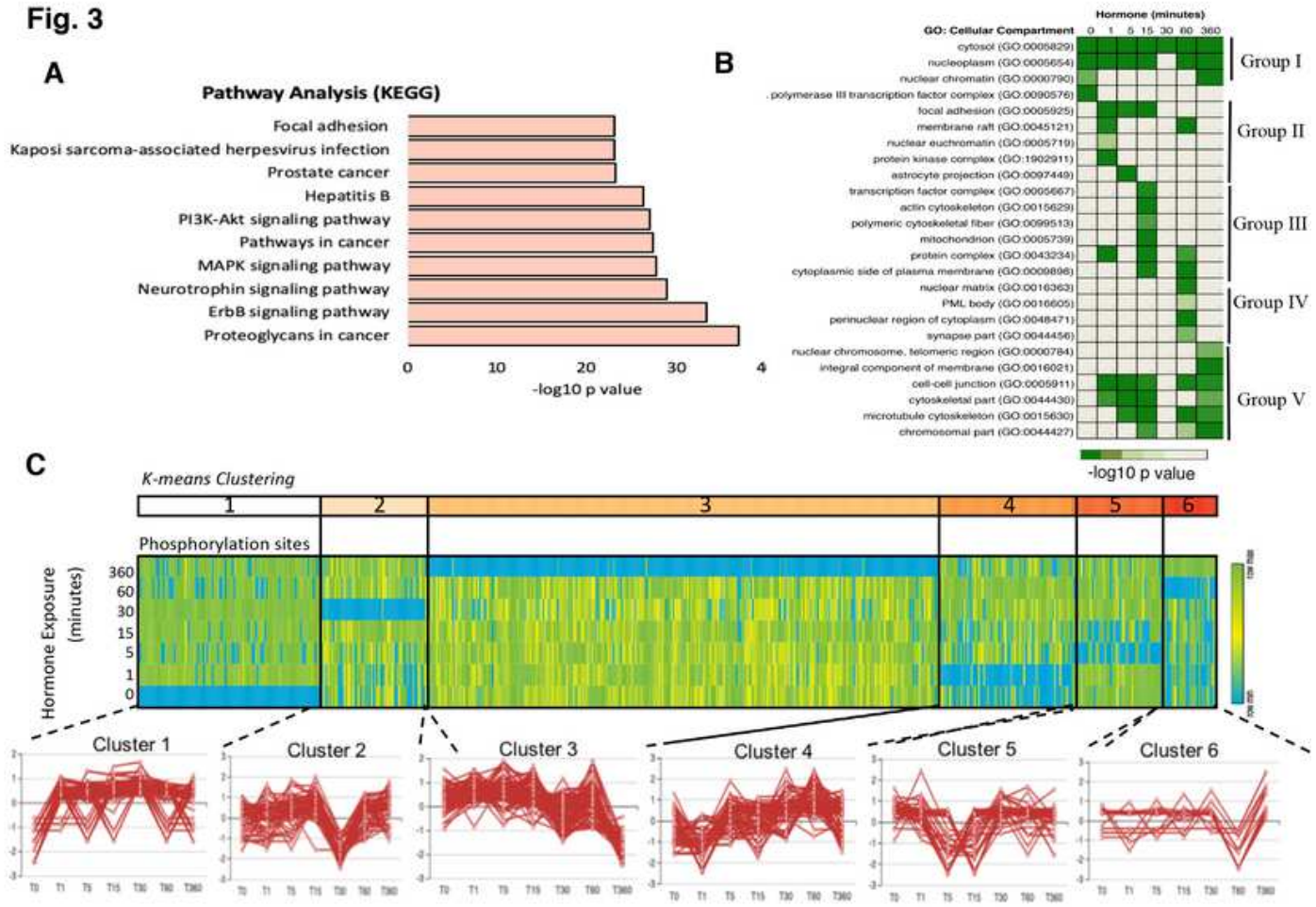

D

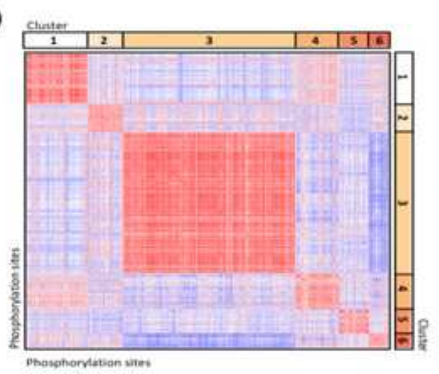

6

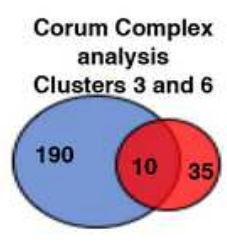

E

GO-Biological process

Cluster 1 and 4: Early

Signal

Regulation

Positive Process
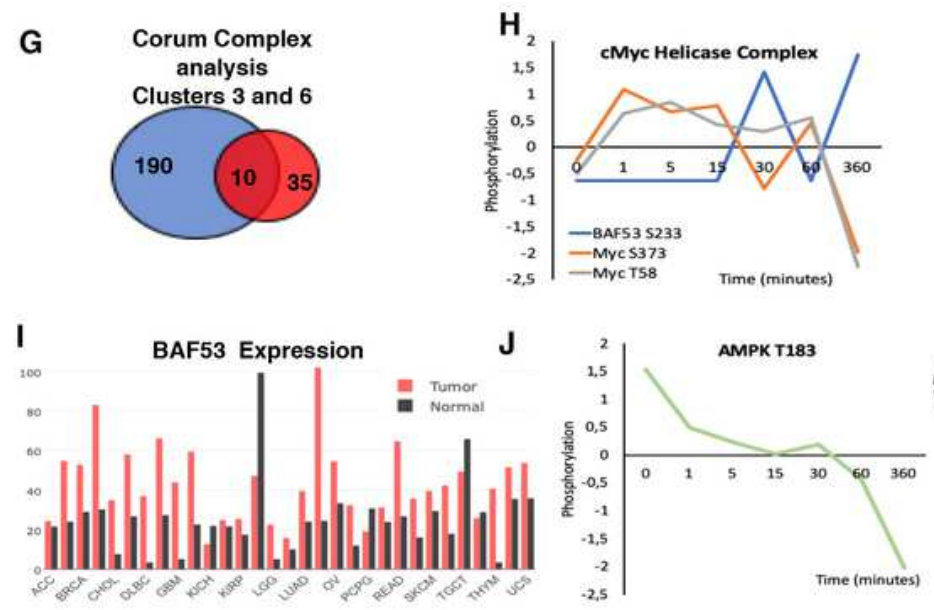

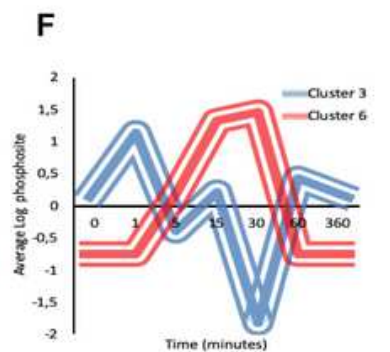

K
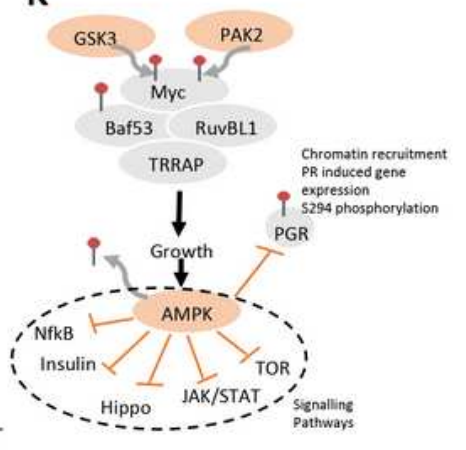

Figure 3 
Combining Target Antibody Arrays and Shotgun Phosphoproteomic datasets following hormone A) KEGG pathway enrichment analysis of proteins identified as regulated by phosphorylation in response to hormone. B) Cellular component analysis of phosphosites enriched per time point. Showing the hormone induced phosphorylation of the nucleoplasm and cytosol across all time points (group I) the activation of membrane raft proteins enriched at 1 minute (Group II) and phosphorylation of mitochondrial proteins enriched at 15 minutes (Group III), activation of nuclear structures; PML bodies and the nuclear matrix at 60 minutes (Group IV) and the activation of the cell-cell junctions and microtubules at 360 minutes (Group V). C) K mean clustering of all significantly regulated phosphorylation sites over time reveals 6 distinct clusters. D) Similarity matrix of clusters 1-6 reveals similar dynamics for clusters 1 and 4 and an opposing similarity in phosphorylation dynamics for clusters 3 and 6 . Red indicates highly similar, well correlated, blue inversely correlated patterns of regulation. E) Word cloud showing the enrichment of GObiological processes associated with proteins identified in similar clusters 1 and 4 "Early risers" which are regulated rapidly after hormone. F) Graph showing the opposing phosphorylation dynamic of proteins within clusters 3 and 6 . G) Venn diagram showing the overlap of significantly identified Corum protein complexes identified in clusters 3 and 6. H) Phosphorylation dynamic in response to hormone of Myc S373, and T58 and BAF53 S233. I) Expression level of BAF53 in tumour versus normal tissue within the TGCA dataset. J) Phosphorylation of AMPL T183 decreases rapidly in response to hormone. K) Model showing the key role of AMPK dephosphorylation in response to hormone in breast cancer cells, AMPK dephosphorylation is required in order for subsequent signaling cascades including NFkB, insulin, Hippo, JAK/STAT and mTOR to continue and the phosphorylation of PR S294 to take place. 
Fig. 4

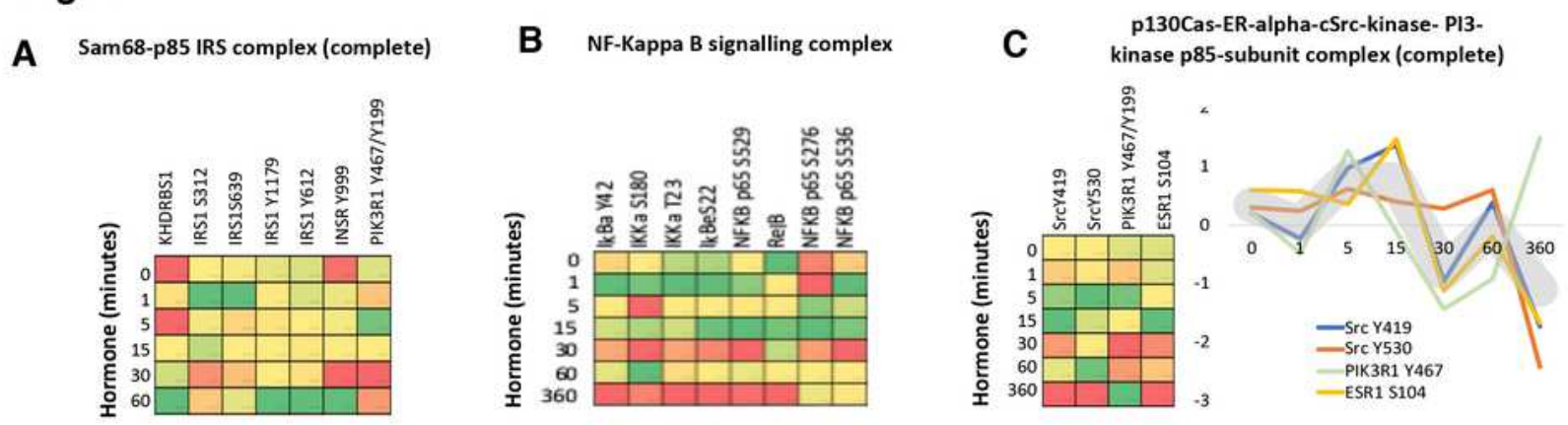

D

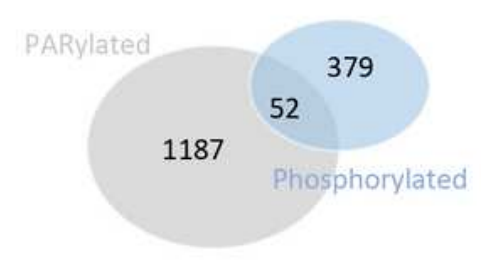

E

F
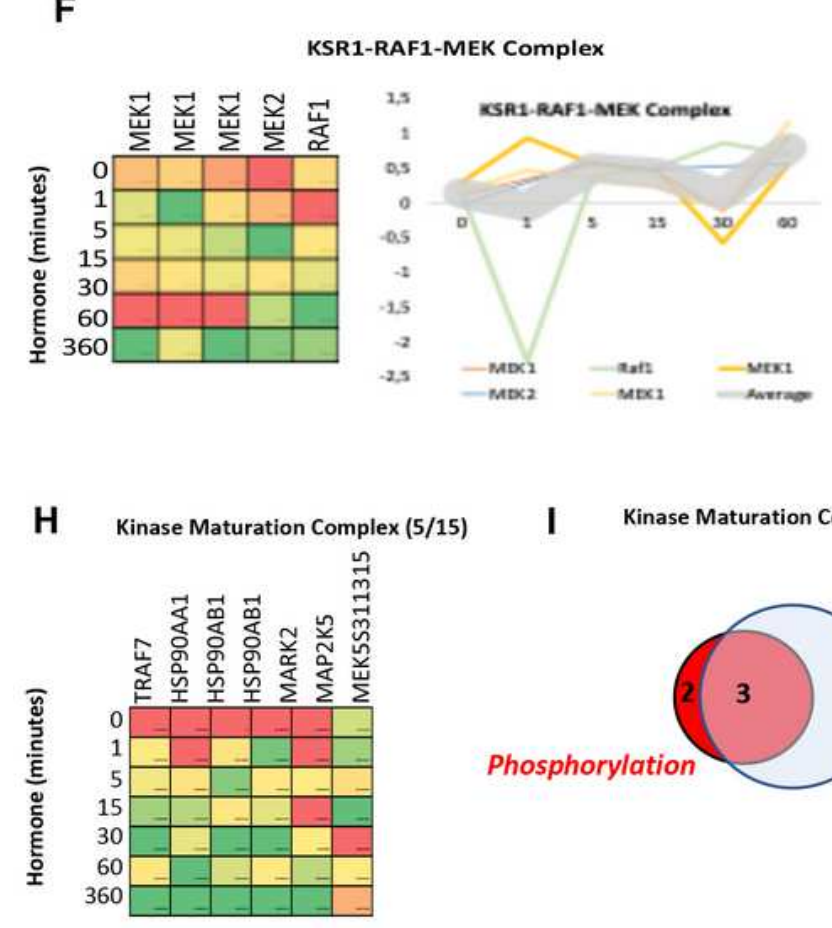
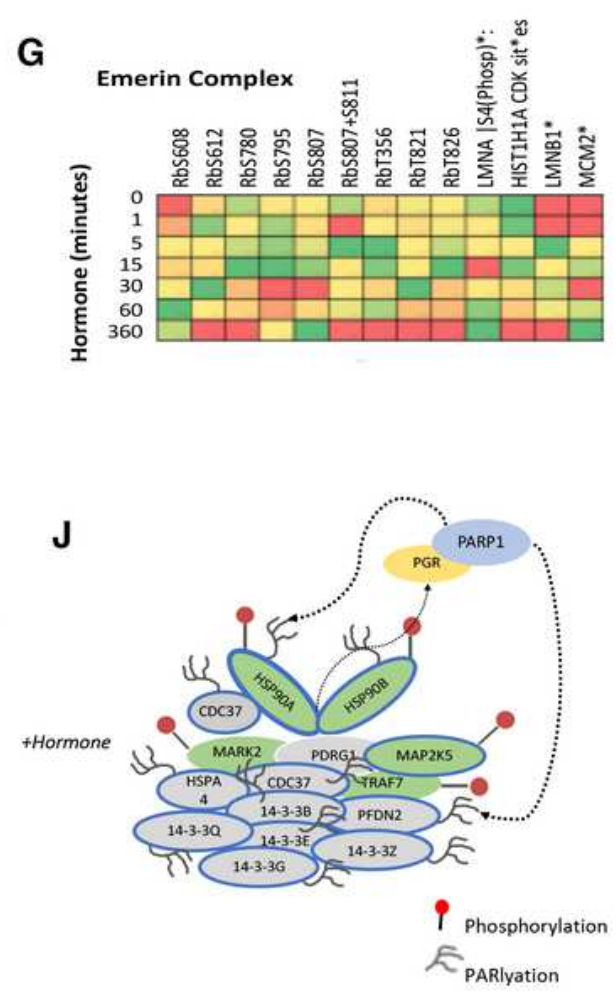

\section{Figure 4}

Protein Complex analysis and Overlap of PARylation and Phosphorylation in response to Progesterone. Heatmaps showing the phosphorylation of proteins within the Sam68-p85 IRS (A) and NF-kappa B (B) signalling complexes in response to hormone over time. C) Heatmap showing the phosphorylation of proteins of the p130 Cas-ER-Src-PI3K complex in response to hormone over time, the coordinated phosphorylation of each phosphosite individually is represented as a line graph (right panel). D) Venn 
diagram showing the overlap of proteins which contain either a phosphorylation site (379), PARylation site (1187) or both PTMs within the same protein after hormone exposure in breast cancer cells (52). E) Word cloud representation showing the GO-cellular component enrichment analysis of the 52 proteins identified as phosphorylated and PARylated in response to hormone (Fig. 4D). F) Heatmap showing the phosphorylation of components of the KRS1-RAF1-MEK signalling complex in response to hormone over time, all proteins shown are phosphorylated and PARylated and the dynamics of individual sites is shown on the right panel. G) Heatmap showing the phosphorylation proteins of the Emerin complex in response to hormone over time. H) Heatmap showing the phosphorylation of proteins (5/15) within the Kinase Maturation complex in response to hormone over time, phosphorylation dynamics of individual sites is shown (lower panel). I) Venn diagram showing the phosphorylation and or PARylation of proteins contained within the kinase maturation complex; $14 / 15$ protein components of the complex contain at least one of the PTMs. J) Schematic representation of the complex components, PARylated proteins are indicated by blue circle, Phosphorylated by red (right panel) 
Fig. 5

A
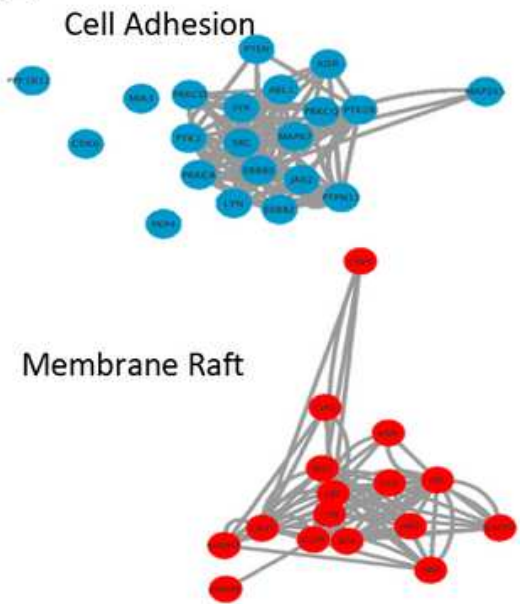

C

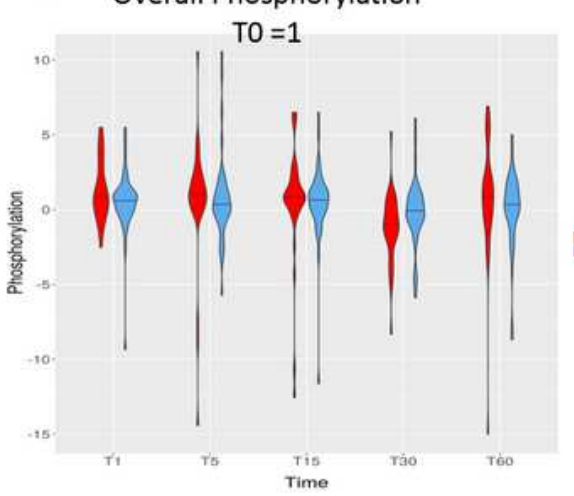

$\mathbf{E}$

Cell Adhesion-Membrane Raft-Cytoskeleton regulation Hormone (Time minutes)

\begin{tabular}{|c|c|c|c|c|}
\hline \multirow{2}{*}{\multicolumn{5}{|c|}{ Membrane Raft 1}} \\
\hline & & & & \\
\hline & & & & \\
\hline & & & & \\
\hline
\end{tabular}

B

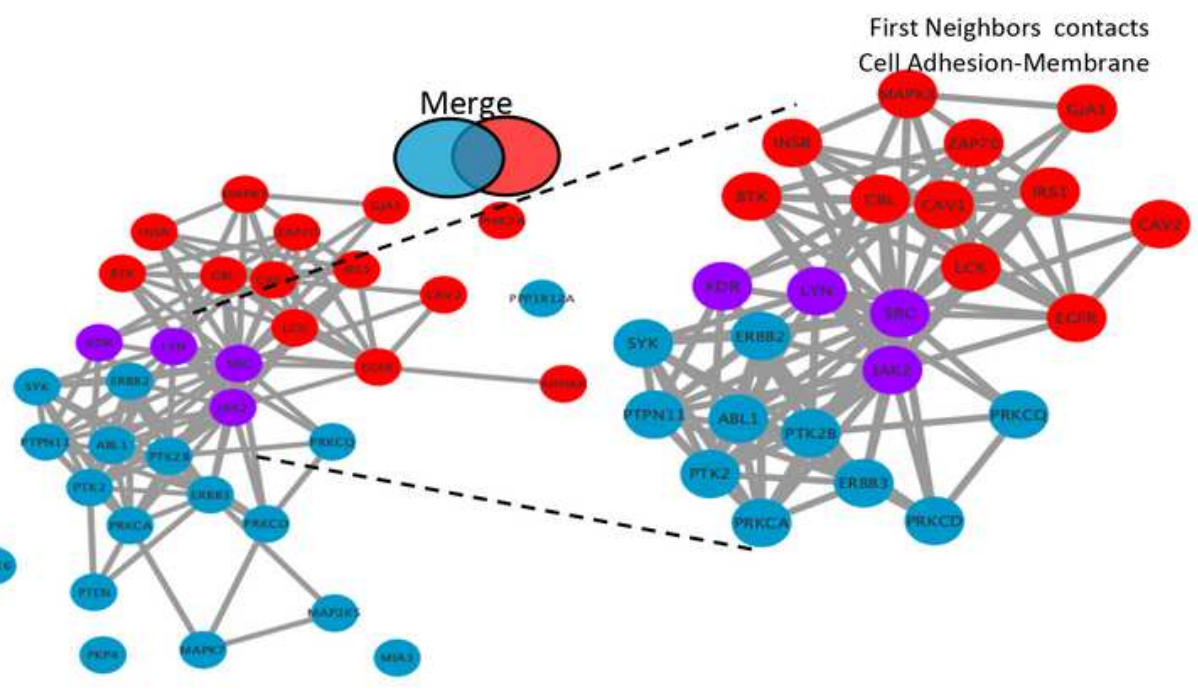

D
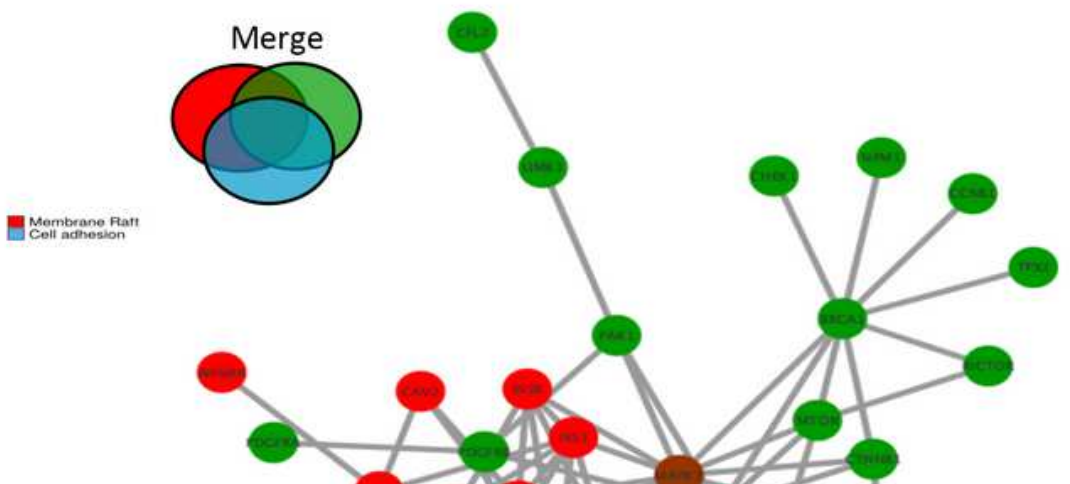

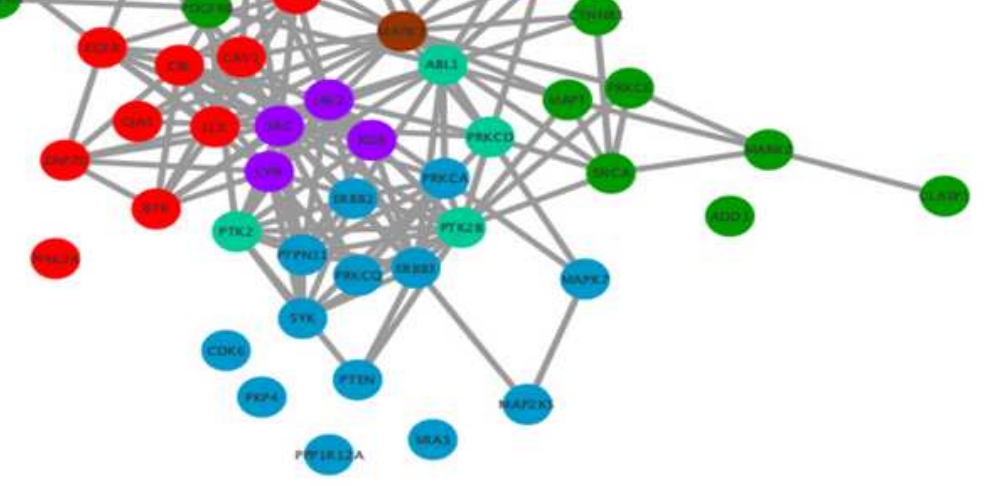

\section{Figure 5}

Combining PPI networks from distinct cellular compartments reveals a coordinated crosstalk. A) PPI network showing the significantly regulated phosphorylated proteins located in cell adhesion (blue) and the membrane raft (red) identified in response to hormone. B) Merge of the cell adhesion network (Fig. 5A, blue) and membrane raft network (Fig. 5A red). The two networks connect based on known PPI however no protein was identified as annotated in both sets. This integration of the two networks is highlighted 
(right panel) where proteins from each network were selected based on having a first neighbour with a protein of the other network. C) Violin plot showing the average phosphorylation of proteins over time in response to hormone within the membrane raft or cell adhesion networks. Data is normalised to time $0=1$. D) Merge of Cell Adhesion Membrane (Fig. 6B) and the cytoskeleton networks. The two networks are merged based on known PPI. Proteins annotated in more than one function are coloured based on the Venn diagram (i.e. cytoskeleton and cell Adhesion; light green, membrane raft and cytoskeleton; brown). E) Heatmap showing the average phosphorylation of all proteins within each network in response to hormone over time, showing the activation of the membrane raft first at 1 minute followed by the cytoskeleton and cell adhesion. 
Fig. 6

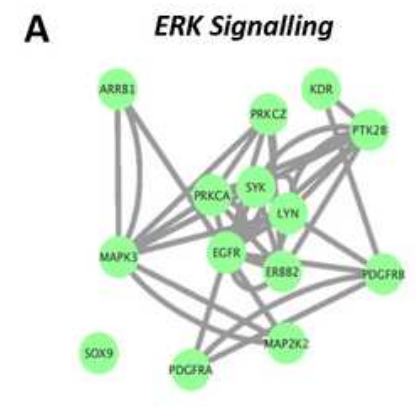

C
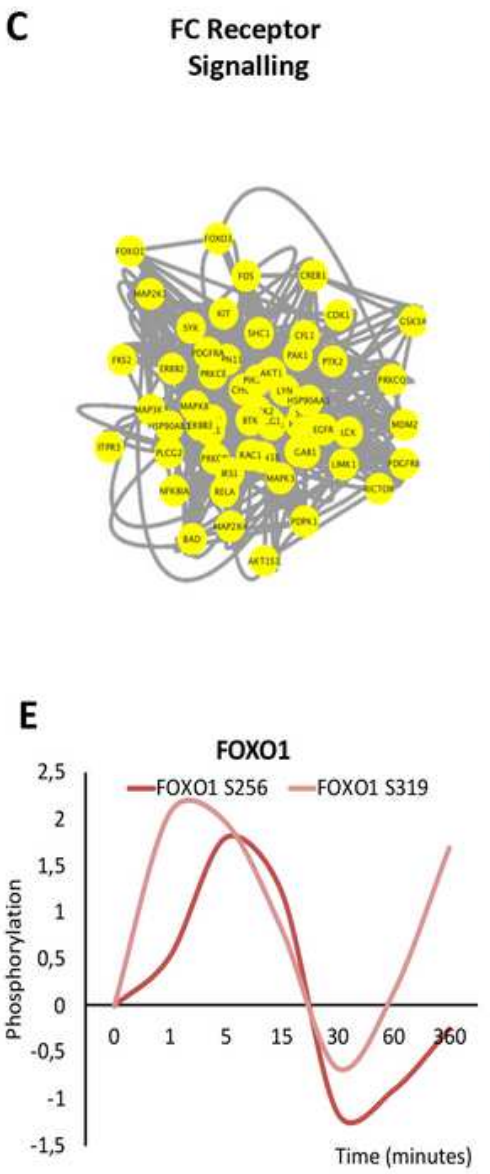

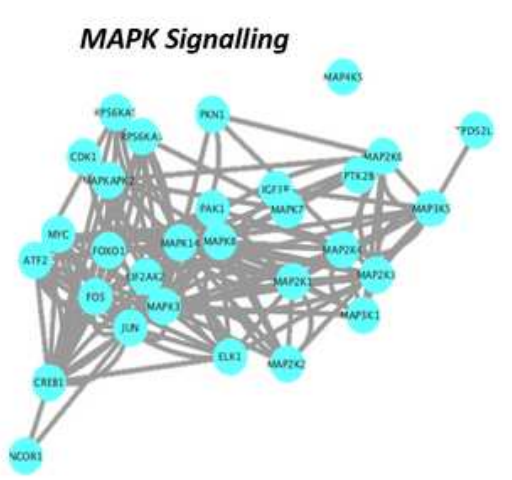

B
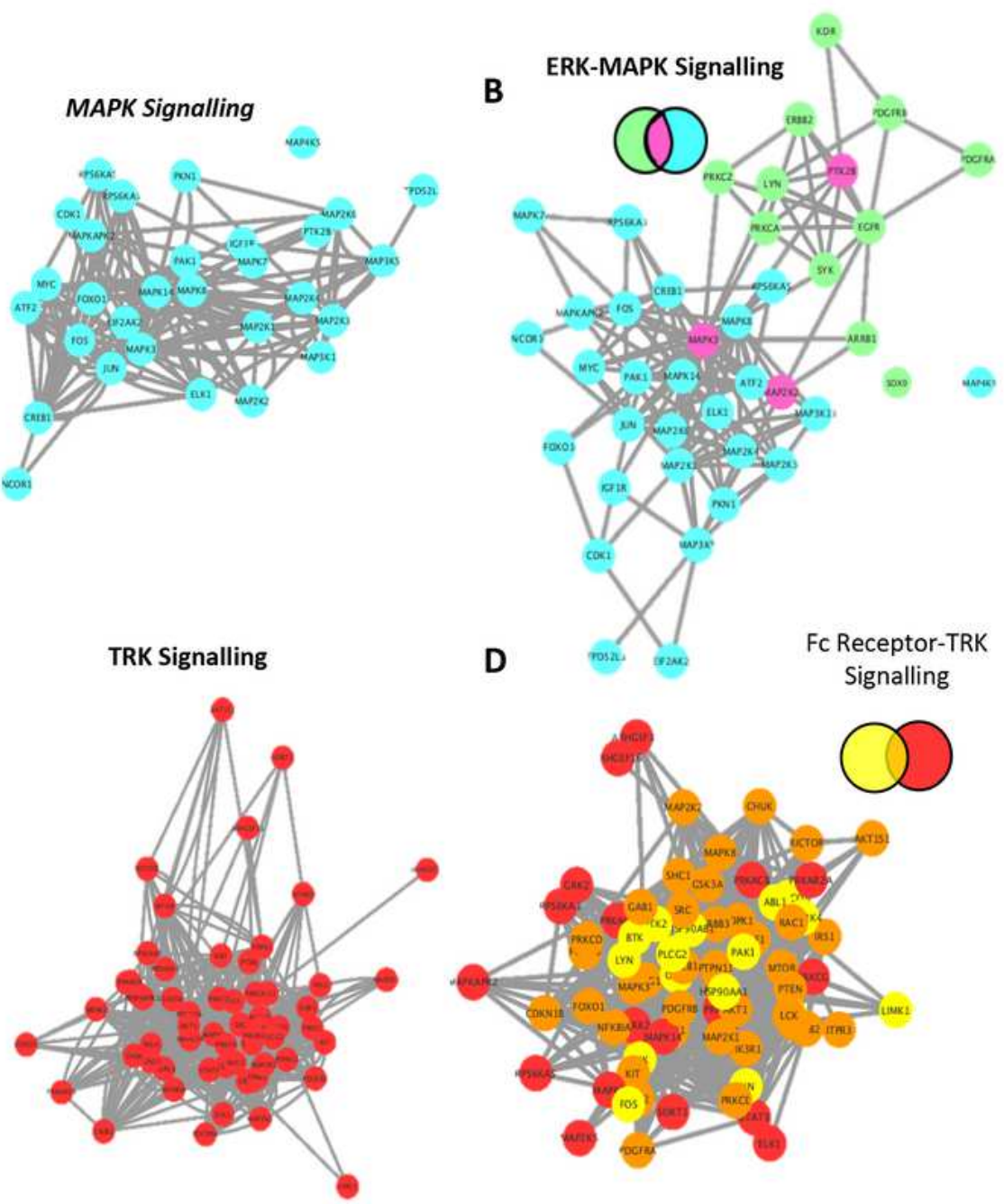

G

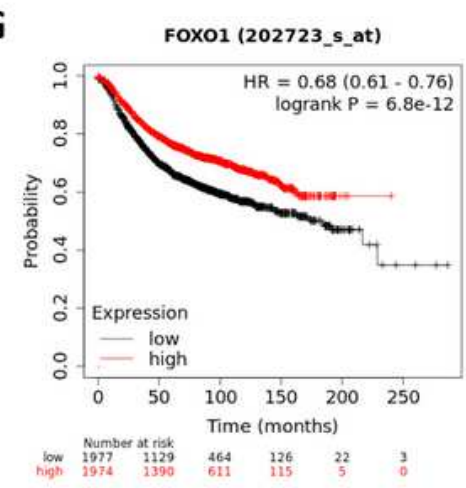

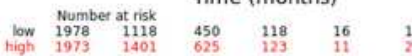

\section{Figure 6}

Network Integration of signalling networks identified in response to hormone. A) PPI network showing the phosphorylated proteins present within the ERK signalling cascade (green) and the MAPK cascade (blue) identified in response to hormone. B) Merge of ERK-MAPK networks (Fig. 6A). The two networks are merged based on known PPI. Proteins annotated in both pathways are coloured based on the Venn diagram (fuchsia). C) PPI network showing the phosphorylated proteins present within the FC-receptor 
(yellow) and TRK-neurorophin (red) signalling pathways (yellow) identified in response to hormone (left and middle panel). D) Merge of FC-receptor and TRK neurotrophin networks. The two networks are merged based on known PPI. Proteins annotated in both pathways are coloured based on the Venn diagram (orange). E) Rapid and coordinated phosphorylation of FOXO1 S256 and FOXO S319 in 1267 response to hormone. Kaplan Meyer overall survival of patients stratified based on the expression of NTRK2 ( $F$ ) and FOXO1 (G) in breast cancer patients ( $\mathrm{p}=9.4 \mathrm{E}-10$ and 6.8e-12 respectively). All networks, PPIs and integrated cascades are supplied in Cytoscape session 2.

\section{Supplementary Files}

This is a list of supplementary files associated with this preprint. Click to download.

- SuppTable7.xlsx

- SuppTables1and2.xIsx

- SuppTables3and4.xlsx

- SuppTables810.xIsx

- Supptable5and6.xlsx

- SupplementaryFigures1to3.pdf

- SupplementaryFigures4to7.pdf 